\title{
The Riemannian Geometry of Physical Systems of Curves (*).
}

\author{
by John DE Crcco (a Chicago, U S.A.)
}

To Enrico Bompiani on his scientific Jubilee

Summary. - The properties of a physical system $S_{k}$ vhere $k \neq-1$, of $\infty^{2 n-1}$ trajectories C. in a Riemannian space $V_{n}$ are developed. The intrinsic differential equations and the equations of Lagrange, of a physical system $S_{k}$, are derived. The Lagrangian function $I$ and the Hamiltonian function $H$, are studied in the conservative case. Also included are systems of the type $(G)$, cuvvature trajectories, and natural families. The Appell transformation $T$ of a dynamical system $S_{0}$ in a Riemannian space $\nabla_{n}$, is obtained. Finally, contact transformations and the transformation theory of $a$ physical system $S_{k}$ where $k \neq-1$, are considered in detail.

1. Physical systems $S_{k}$. Consider a field of force whose force vector acting at any point $x=x^{i}$ of a Riemannian space $V_{n}$, is $\Phi=\Phi^{i}=\Phi_{i}$. The force vector $\Phi$, is considered to be a vector function of position only, and to be class two throughout a certain region of $V_{n}$. Therefore, each of the contravariant components $\Phi^{i}$ or of the covariant components $\Phi_{i}$ of the force vector $\Phi$, is a continuous function of $x=\left(x^{1}, x^{2}, \ldots, x^{n}\right)$, and possesses continuous partial derivatives of the first and second orders with respect to the $x^{i}$, throughout the given region of $V_{\ldots}$. Such a field of force is said to be a positional field of force $\Phi$ in the Riemannian space $V_{n}$.

We omit from consideration the trivial case where the force vector $\Phi$ is identically zero, in which case the physical eurves under discussion are the $\infty^{2 n-2}$ geodesics of $V_{n}$.

By a path $C$ in $V_{n}$, we shall mean a locus of points $x$ in the region of definition of the field of force $\Phi$, given by the parametric equations $x^{i}=x^{i}(t)$ for $i=1,2, \ldots, \ldots, n$, where the parameter $t$ is considered to be the time $t$ and varies in the closed interval $a \leqq t \leqq b$ with $a<b$. It is understood that each $x^{i}(t)$ is of class three in $a \leqq t \leqq b$. Thus if dots denote differentiations with respect to the time $t$, then each of the 4 n functions $x^{i}(t), \quad \dot{x}^{i}(t), \ddot{x}^{i}(t), \dddot{x}^{i}(t)$ for $i=1,2, \ldots, n$, is a continuous function of the time $t$ in $a \leqq t \leqq b$. Also the condition is imposed that at least one $\dot{x}^{i}(t)$, is not identically zero in $a \leqq t \leqq b$. A path $C$ is called a curve $O$ in $V_{n}$.

(*) KASARR, Differential geometric aspecte of aynamics, The Princeton Colloquium Lectures, 1909. Published by the "American Mathematical Society, Providence, Rhode Island, 1913, and reprinted 1934. 
A physicat system $S_{k}$, or more simply, a system $S_{k}$, of $\infty^{2 n-1}$ curves in the given positional field of force $\Phi$ of the Riemannian space $V_{n}$, is composed of curves $C$ along each of which a constrained motion is possible such that the force vector $\Phi$ is tangent to the osculating geodesic surface of the curve $C$ at each point $x$ of $C$, and the pressure $P$, along the principal normal to the curve $C$, is proportional to the normal component $N$ of the force vector $\Phi$. Thus $P=k N$ where $(k \neq-1)$, is the constant factor of proportionality, along each one of the $\infty^{2 n-1}$ curves $C$ of the physical system $S_{k}$.

THEOREM 1.1. - A physical system $S_{k}$ of $\infty^{2 n-1}$ curves $C$ of the Riemannian space $V_{n}$, is given by the system of $n$ ordinary second order differential equations

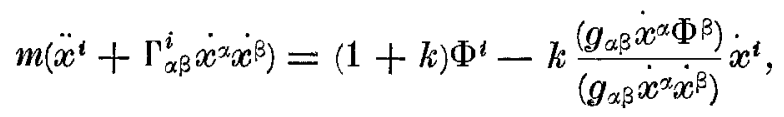

for $i=1,2, \ldots, n$.

In these equations, $m>0$, is the constant mass of the particle traversing the curve $C$. The $g_{i j}$ form the metric symmetric covariant tensor of second order of the Riemannian space $V_{n}$, and the $\Gamma_{\alpha \beta}^{i}$ are the Christoffel symbols of second kind of $V_{n}$.

Of course $v^{2}=g_{\alpha \beta} \dot{x}^{\alpha} \dot{x}^{\beta}>0$, along any curve $C$ of the physical system $S_{k}$.

It is assumed that each one of the $g_{i j}$ is of the class three throughout the given of $V_{n}$. Then the $\Gamma_{\alpha \beta}^{i}$ are all of elass two in this region of $V_{n}$.

From the equations (1.1), it is deduced that each $x^{i}(t)$ of the curve $C$ of a physical system $S_{k}$, is at least of class four in $a \leqq t \leqq b$.

The important special cases of physical interest of a physical system $S_{k}$ are the four following ones.

(a) For $k=0$, this is the system $S_{0}$ of dynamical trajectories. These are the paths of motion of a particle due to the influence of the given field of force $\Phi$.

(b) For $k=1$, this is the system $S_{1}$ of general catenaries. These are the curves in the reversed field of force $-\Phi$, formed from the resulting equilibrium where an inextensible flexible homogeneous string is suspended from two distinct points in the given region.

(c) For $k=-2$, this is the system $S_{-2}$ of general brachistochrones. If the field of force $\Phi$ is conservative, the system $S_{-2}$ is composed of true brachistochrones. In this case, these are the eurves along which the time of constrained motion between any two distinct points is least. Whether or 
not the field of foree $\Phi$ is conservative, we call the curves of a system $S_{-2}$, general brachistochrones.

(d) For $k=\infty$, this is the system $S_{\infty}$ of velocity curves. Of course, $S_{\infty}$ is the limit of a system $S_{k}$ as $k$ becomes infinite. A velocity curve $C$ is one to which there corresponds a fixed speed $v_{0}>0$, such that a particle, starting from any lineal element $E$ of $C$ with the speed $v_{0}$, describes a dynamical trajectory which initially osculates the curve $C$.

Physical systems of curves have been studied by Kasner and De Croco, on a Euclidean plane, in Euclidean space, and on a surface of Euclidean space [1]. In the present article, we shall give extensions of some of these theorems to a Riemannian manifold $V_{n}$. In the case of a conservative field of force, a discussion is given for a physical system $S_{k}$ of the equations of Lagrange, the infinitesimal contact transformation of mechanics studied extensively by Lie and Vessiot, and certain related dynamical theorems of KASNer. Finally some theorems are obtained for arbitrary positional fields of force whether conservative or not.

2. Preliminary formulas. - Consider a Riemannian manifold $V_{n}$ where the square of the differential $d s$ of arc length $s$ of a curve $C$, is given by the positive definite quadratic differential form

$$
d s^{2}=g_{i j} d x^{i} d x^{j}
$$

The $g_{i j}$ form the metric symmetrie covariant tensor of second order with $g=\left|g_{i j}\right|>0$. For this $V_{n}$, the Christoffel symbols of the first and second kinds, are

$$
\begin{aligned}
& \Gamma_{i j ; k}=\frac{1}{2}\left(\frac{\partial g_{i k}}{\partial x^{j}}+\frac{\partial g_{j k}}{\partial x^{i}}-\frac{\partial g_{i j}}{\partial x^{k}}\right), \\
& \Gamma_{j k}^{i}=g^{i l} \Gamma_{j k ; l} .
\end{aligned}
$$

The unit tangent vector of a curve $C$ in $V_{n}$ is $d x^{i} / d s$. The vector curvature $x$ of this curve $C$, is

$$
x=x^{i}=\frac{d^{2} x^{i}}{d s^{2}}+\Gamma_{\alpha \beta}^{i} \frac{d x^{\alpha}}{d s} \frac{d x^{\beta}}{d s} .
$$

If $|x|^{2}=g_{i j} x^{i} x^{i}>0$, at a point $x$ of the curve $C$, its curvature is $|x|>0$, at this point $x$ of $C$. Then its radius $r$ of curvature at this point $x$, is $r=1 /|x|$. 
At a point $x$ of the curve $C$, the unit tangent vector $\frac{d x^{i}}{d s}$ and the unit (principal) normal vector $x^{i} /|x|=r x^{i}$, when $|x|>0$, are orthogonal.

Consider a point $x$ of the curve $C$ for which $|x|=1 / r>0$. The pencil of vectors spanned by the unit tangent vector $\frac{d x^{i}}{d s}$ and the unit normal vector $r x^{i}$, is composed of all vectors of the form

$$
a \frac{d x^{i}}{d s}+b r x^{i}
$$

where $a$ and $b$ are arbitrary scalars. If at least one of the two scalars $a$ and $b$ is not zero, there is a unique geodesic defined by $x^{i}=0$, touching a given one of such vectors. The resulting totality of $\infty^{1}$ geodesics describe the osculating geodesic surface at the given point $x$ of the curve $C$.

Let a particle of mass $m>0$, traverse a given curve $C: x^{i}=x^{i}(t)$, for $i=1,2, \ldots, n$ of the Riemannian space $V_{n}$. The velocity vector of this particle is $V=\dot{x}^{i}$, and its speed is $v=\left(g_{i j} \dot{x}^{i} x^{j}\right)^{\frac{1}{2}}>0$. The acceleration vector $a$ of this particle, is

$$
a^{i}=\ddot{x^{i}}+\Gamma_{\alpha \beta}^{i} \dot{x}^{\alpha} \dot{x}^{\beta} ; \text { or } a_{i}=g_{i j} \ddot{x}^{j}+\Gamma_{\alpha \beta, i} \dot{x}^{\alpha} \dot{x}^{\beta} \text {. }
$$

The first is the contravariant form $a^{i}$, and the second is the covariant form $a_{i}$ of the acceleration vector $a$. The magnitude of $a$ is $|a|=\left(g_{i j} a^{i} a^{j}\right)^{\frac{1}{2}}$.

The kinetic energy $T$ of a particle of mass $m>0$, traversing $a$ curve $C$ in $V_{n}$, is

$$
T=\frac{m v^{2}}{2}=\frac{m}{2} g_{i j} \dot{x}^{i} \dot{x}^{j}
$$

By the preceding formulas, it is evident that

$$
m a_{i}=\frac{d}{d t}\left(\frac{\partial T}{\partial \dot{x}^{i}}\right)-\frac{\partial T}{\partial x^{i}}
$$

Let $a_{t}$ and $a_{n}$ denote the tangential and normal components of the acceleration vector $a$. These are given by the equations

$$
a_{t}=\dot{v}=v \frac{d v}{d s}, \quad a_{n}=\frac{v^{2}}{r} .
$$


The acceleration vector $a$ is tangent to the osculating geodesic surface of the curve $C$ at the point $x$ of $C$. Then

$$
a^{i}=a_{t} \frac{d x^{i}}{d s}+a_{n} r x^{i}=\dot{v} \frac{d x^{i}}{d s}+v^{2} x^{i}
$$

Therefore

$$
v^{2} x^{i}=a^{i}-\frac{\dot{v}}{v} \dot{x}^{i}
$$

3. Elementary properties of an arbitrary positional field of force. Consider a curve $C$ in $V_{n}$ such that for every point $x$ of $C$, its vector geodesic curvature $x$ is not the zero vector. Let $T_{1}$ and $N_{1}=N$, denote the tangential and normal components of the force vector $\Phi$ relative to this curve $C$. Then

$$
T_{1}=g_{\alpha \beta} \Phi^{\alpha} \frac{d x^{\beta}}{d s}=\Phi_{\alpha} \frac{d x^{\alpha}}{d s}, N_{1}=N=r g_{\alpha \beta} \Phi^{\alpha} \varkappa^{\beta}=r \Phi_{\alpha} \chi^{\alpha}
$$

If a particle of mass $m>0$, traverses this curve $C$, then its pressure $P$ along the curve $C$ is

$$
P=\frac{m v^{2}}{r}-N=\frac{m v^{2}}{r}-r g_{\alpha \beta} \Phi^{\alpha_{\chi} \beta}=\frac{m v^{2}}{r}-r \Phi_{\alpha} \chi^{\alpha} .
$$

The space derivative $\frac{D \Phi}{d s}$ of the force $\Phi$, is its absolute derivative along the curve $C$ with respect to arc length $s$. The contravariant and covariant forms of this space derivative $\frac{D_{\Phi}}{d s}$, are

$$
\begin{aligned}
& \frac{D \Phi^{i}}{d s}=\frac{d \Phi^{i}}{d s}+\Gamma_{\alpha \beta}^{i} \Phi^{\alpha} \frac{d x^{\beta}}{d s} \\
& \frac{D \Phi_{i}}{d s}=\frac{d \Phi_{i}}{d s}-\Gamma_{i \beta}^{\alpha} \Phi_{\alpha} \frac{d x^{\beta}}{d s}
\end{aligned}
$$

Let $T_{2}$ and $N_{2}$ denote the tangential and normal components along the curve $C^{\prime}$ of the space derivative $\frac{D \Phi}{d s}$, with respect to the arc length $s$, of the force vector $\Phi$, along this same curve $C[2]$. Then

$$
T_{2}=g_{\alpha \beta} \frac{D \Phi^{\alpha}}{d s} \frac{d x^{\beta}}{d s}=\frac{D \Phi_{\alpha}}{d s} \frac{d x^{\alpha}}{d s}, \quad N_{2}=r g_{\alpha \beta} \frac{D \Phi^{\alpha}}{d s} x^{\beta}=r \frac{D \Phi_{\alpha}}{d s} x^{\alpha}
$$


It is clear that

$$
\frac{d T_{1}}{d s}=T_{2}+\frac{N}{r}
$$

Also it seen that

$$
\frac{d N}{d s}=\frac{d N_{1}}{d s}=N_{2}+\frac{N}{r} \frac{d r}{d s}+r \Phi_{\alpha} \frac{D x^{\alpha}}{d s}
$$

This can be written in the from

$$
\frac{d N}{d s}=\frac{d N_{1}}{d s}=N_{2}+\Phi_{\alpha} \frac{D\left(r x^{\alpha}\right)}{d s} .
$$

Consider a fixed point $x$ of the curve C. [3]. The first principal unit vector $\tau_{1}$, is the unit tangent vector $\tau_{1}=\frac{d x}{d s}$. The second principal unit vector $\tau_{2}$ or the first principal unit normal vector $\tau_{2}$, is the unit vector in the direction of the vector geodesic curvature so that $\tau_{2}=r \chi$. The scalar $r=r_{1}$, is the first radius of curvature. Then

$$
\frac{D \tau_{1}}{d s}=x=\frac{1}{r_{1}} \tau_{2}
$$

At the fixed point $x$ of the curve $C$, the third principal unit vector $\tau_{3}$, or the second principal unit normal vector $\tau_{3}$, and the second radius $r_{2}$ of curvature, are defined by the vector equation

$$
\frac{D \tau_{2}}{d s}=-\frac{1}{r_{1}} \tau_{1}+\frac{1}{r_{2}} \tau_{3}
$$

It may be that $\frac{1}{r_{2}}=0$, in which ease $\tau_{3}$, is any unit vector if it exists which is orthogonal to $\tau_{1}$ and $\tau_{2}$.

Suppose that the curve $C$ is of class $(i+1)$ in the closed interval $a \leq t \leqq b$ with $a<b$, where $i \geqq 2$. The principal unit vector $\tau_{i_{+1}}$ of order $(i+1)$, or the principal unit normal vector $\tau_{i+1}$ of order $i$, and the radius $r_{i}$ of curvature of order $i$, at the fixed point $x$ of the curve $C$, are given by the Serret-Frenet formulas

$$
\frac{D \tau_{i}}{d s}=-\frac{1}{r_{i-1}} \tau_{i-1}+\frac{1}{r_{i}} \tau_{i+1}
$$


where $i=.1,2, \ldots, n$. Here it is understood that $\frac{1}{r_{0}}=0$, and $\frac{1}{r_{n}}=0$. It may be that $\frac{1}{r_{i}}=0$, in which ease $\tau_{i+1}$, is any unit vector if it exists, which is orthogonal to $\tau_{1}, \tau_{2}, \ldots, \tau_{i}$.

In general, a curve $C$ at a fixed point $x$, possesses $(n-1)$ radii of curvature, namely, $r_{1}, r_{2}, \ldots, r_{n-1}$.

By (3.8) and (3.9), it is found that $(3.7)$ can be written in the form

$$
\frac{d N}{d s}=\frac{d N_{1}}{d s}={ }^{n} N_{2}-\frac{T}{r}+\frac{1}{r_{2}} \Phi_{\alpha} \tau_{2}^{\alpha}
$$

THeOREM 3.1 - At a fixed point $x$ of a curve $C$ of a Riemannian space $V_{n}$, the first variations $\frac{d T_{1}}{d s}$ and $\frac{d N}{d s}=\frac{d N_{1}}{d s}$, of the tangential component $T_{1}$ and the normal component $N=N_{1}$, of the force vector $\Phi$, are given by the two expressions

$$
\frac{d T_{1}}{d s}=T_{2}+\frac{N}{r}, \frac{d N}{d s}=N_{2}-\frac{T_{1}}{r}
$$

if and only if either $\frac{1}{r_{2}}=0$, or the force vector $\Phi$ is orthogonal to the third unit principal vector $\tau_{3}$, at this point $x$ of the curve $C$. In particular, these two formulas are valid if the force vector $\Phi$ is tangent to the osculating geodesic surface at this point $x$ of the curve $C$.

This proposition follows from equations (3.5) and (3.11).

As a particle of mass $m>0$, traverses a curve $C$ from a fixed point $x_{0}$ to a variable point $x$, then the work $W$ of the particle is given by the line integral

$$
W=\int_{x_{0}}^{x} T_{1} d s=\int_{x_{0}}^{a} \Phi_{\alpha} d x^{\alpha}
$$

If a particle of mass $m>0$, is constrained to move along a given curve $C$ from a fixed point $x_{0}$ to a fixed point $x$, then

$$
m \dot{v}=m v \frac{d v}{d s}=T_{1}=\Phi_{\alpha} \frac{d x^{\alpha}}{d s}
$$

Therefore

$$
\frac{m v^{2}}{2}-\frac{m v_{0}^{2}}{2}=\int_{x_{0}}^{\infty} \Phi_{\alpha} d x^{\alpha}=W
$$


This states that the work $W$ of the particle is equal to the change in its kinetic energy

A Faraday line of force $C$ of the given field of force $\Phi$ is one such that the force vector $\Phi$ is tangent to the curve $C$ at every point $x$ of $C$. Evidently $N=N_{1}=0$, and $T_{1}=|\Phi|$, along a line of force $C$.

The system of $n$ ordinary first order differential equations defining the lines of force $C$, is

$$
\frac{d x^{i}}{d s}=\frac{\Phi^{i}}{|\Phi|}
$$

for $i=1,2, \ldots, n$. There are $\infty^{n-1}$ Farady lines of force in an arbitrary positional field of force $\Phi$.

The equations (3.12) along a line of force $C$, are

$$
\frac{d T_{1}}{d s}=T_{2}, \quad O=N_{2}-\frac{T_{1}}{r}
$$

Therefore if $N_{2} \neq 0$ at a point $x$ of a line of force $C$, its radius $r$ of curvature at this point $x$, is

$$
r=\frac{T_{1}}{N_{2}}
$$

4. Conservative fields of force. It is assumed that the region of defi. nition of the field of force $\Phi$, is simply connected. The given positional field of force $\Phi$, is said to be conserative if and only if the work $W$ is independent of the path [4].

For a conservative field of force $\Phi$, there exists a potential function $V=V(x)$ which is of class three in the given region, such that

$$
\Phi_{i}=-\frac{\partial V}{\partial x^{i}}
$$

for $i=1,2, \ldots, n$. This states that the force vector $\Phi$, is the negative of the gradient $\frac{\partial V}{\partial x^{i}}$, of this potential function $V$. Any two such potential functions $V$, differ by a constant.

By equations (3.14) and (4.1), the energy equation

$$
T+\nabla=E
$$


is valid in a conservative field of force $\Phi$. In this equation, $T=\frac{m v^{2}}{2}$, is the kinetic energy, $V$ is the potential energy, and $E$ is the constant total energy.

In a conservative field of force $\Phi$, a Riemannian space of dimension $(n-1)$, along which the potential function $V$ is constant, is called a potential surface.

Thcre are $\infty^{1}$ potential surfaces given by the equation

$$
V=V(x)=\mathrm{constant}
$$

The $\infty^{n-1}$ Faraday lines of force of a conservative field of force $\Phi$, are given by the system of $n$ ordinary first order differential equations

$$
\frac{d x^{\prime}}{d s}=-\frac{g^{i s} \frac{\partial V}{\partial x^{j}}}{\left|\frac{\partial V}{\partial x}\right|}
$$

for $i=1,2, \ldots, n$. The $\sim^{n-1}$ Faraday lines of force of a conservative field of force $\Phi$, are orthogonal to the $\infty^{1}$ potential surfaces.

In a conservative field of force $\Phi$, the $\infty^{1}$ Faraday lines of force are geodesies if and only if the magnitude $|\Phi|$ of the force vector $\Phi$, is a function of the potential function $V=V(x)$, alone.

5. The differential equations of a physical system $S_{k}$ where $k \neq-1$, $\infty$ [5]. A physical system $S_{k}$ where $k \neq-1, \infty$, of $\infty^{2 n-1}$ curves in $V_{n}$, is defined by the equations

$$
\begin{gathered}
m \dot{v}=m v \frac{d v}{d s}=T_{1}=g_{\alpha \beta} \Phi^{\alpha} \frac{d x^{\beta}}{d s}=\Phi_{\alpha} \frac{d x^{\alpha}}{d s^{i}} \\
m v^{2}=(1+k) r N \\
\Phi^{i}=T_{1} \frac{d x^{i}}{d s}+r N x^{i}
\end{gathered}
$$

Theorem 5.1. - A physical system $S_{k}$ where $k \neq-1, \infty$, of $\infty^{2 n-1}$ curves $C$, in a Riemannian space $V_{n}$, is given by the equations

$$
\begin{aligned}
& m v \frac{d v}{d s}=g_{\alpha \beta} \Phi^{\alpha} \frac{d x^{\beta}}{d s}, m v^{2}=(1+k) r N \\
& m v^{2} x^{i}=(1+k) \Phi^{i}-(1+k)\left(g_{\alpha \beta} \Phi^{\alpha} \frac{d x^{\beta}}{d s}\right) \frac{d x^{i}}{d s}
\end{aligned}
$$

for $i=1,2, \ldots, n$. 
For, the two systems of equations (5.1) and (5.2), are equivalent.

THeonem 5.2. - Either one of the two systems of $n$ ordinary second order differential equations

$$
\begin{aligned}
& m a^{i}=(1+k) \Phi^{i}-\frac{\left(k g_{\alpha \beta} \Phi^{\alpha} \dot{x}^{\beta}\right)}{\left(g_{\alpha \beta} \dot{x}^{\alpha} \dot{x}^{\beta}\right)} \dot{x}^{i}, \\
& m a_{i}=(1+k) \Phi_{i}-\frac{k\left(g_{\alpha \beta} \Phi^{\alpha} \dot{x}^{\beta}\right)}{\left(g_{\alpha \beta} \dot{x^{\alpha}} x^{\beta}\right)}\left(g_{i \alpha} \dot{x}^{\alpha}\right)
\end{aligned}
$$

for $\mathrm{i}=1,2, \ldots, n$, defines a physical system $S_{k}$ where $k \neq-1, \infty$, of $\infty^{2 n-1}$ curves $C$ in a Riemannian space $V_{n}$.

For, by (2.9) and (5.2), it is seen that

$$
m a^{i}=(1+k) \Phi^{i}-k\left(g_{\alpha \beta} \Phi * \frac{d x^{\beta}}{d s}\right) \frac{d x^{i}}{d s} .
$$

Changing the are length $s$ into the time $t$, the first set of equations (5.3), is found.

The first set of equations (5.3), is the contravariant form of such a physical system $S_{k}$. The second set of equations (5.3), is the covariant form of such a physical system $S_{k}$.

It is remarked that Theorem 1.1, is an expanded form of Theorem 5.2.

TheOREM 5.3. - The equations of Lagrange for a physical system $S_{k}$ where $k \neq-1, \infty$, in a Riemannian space $V_{n}$, are

$$
\frac{d}{d t}\left(\frac{\partial T}{\partial \dot{x}^{i}}\right)-\frac{\partial T}{\partial x^{i}}-(1+k) \Phi_{i}+\frac{k}{2 T}\left(g_{\alpha \beta} \Phi^{\alpha} \dot{x}^{\beta}\right) \frac{\partial T}{\partial \dot{x}^{i}}=0
$$

for $i=1,2, \ldots, n$.

This result follows from equations (2.5), (2.6), and (5.3).

For $k=0$, the equations (5.5) become the well-known equations of Lagrange for a dynamical system $S_{0}$.

Upon eliminating the speed $v$ from the equations (5.2), it is found that

$$
\frac{d}{d s}(r N)=\frac{2}{(1+k)} g_{\alpha \beta} \Phi^{x} \frac{d x^{\beta}}{d s}
$$

$$
r N x^{i}=\Phi^{i}-\left(g_{\alpha \beta} \Phi^{\alpha} \frac{d x^{\beta}}{d s}\right) \frac{d x^{i}}{d s}
$$


J. De Crcco: The Riemannian Geometry Of Physical Systems, etc. 349

TheOREM 5.4. - Along a curve $O$ of a physical system $S_{k}$ where $k \neq-1, \infty$, of a Riemannian space $V_{n}$, the first variations $\frac{d r}{d s}$ and $\frac{d N}{d s}$ of the radius $r$ of curvature and of the normal component $N$ of the force vector $\Phi$, are

$$
\begin{aligned}
& N \frac{d r}{d s}=\left(\frac{3+k}{1+k}\right) T_{1}-r N_{2}, \\
& r \frac{d N}{d s}=-T_{1}+r N_{2} .
\end{aligned}
$$

This is deduced from equations (3.12) and (5.6).

THeOREM 5.5. - The intrinsic differential equations of the $\infty^{2 n-1}$ curves $C$ of a physical system $S_{k}$ where $k \neq-1, \infty$, of a Riemannian space $V_{n}$, are

$$
\begin{aligned}
& N \frac{d r}{d s}=\left(\frac{3+k}{1+k}\right) T_{1}-r N_{2}, \\
& r \frac{d N}{d s}=-T_{1}+r N_{2}, \\
& r N x^{i} \Phi^{i}=\Phi^{i}-T_{2} \frac{d x^{i}}{d s},
\end{aligned}
$$

for $i=1,2, \ldots, n$.

This proposition is a consequence of equations (5.6) and (5.7).

The three systems of differential equations (5.2), (5.3), and (5.5), are equivalent. This means that any integral solution of any one of these three systems, is an integral solution of the other two systems. Such an integral solution is called an actual trajectory $C$ of the physical sistem $S_{k}$. Every actual trajectory $O$ of the physical system $S_{k}$, satisfies the intrinsic differential equations (5.8). However, there do exist curves $C$ which obey the intrinsic differential equations (5.8) but which obey neither one of the three systems of differential equations (5.2), (5.3), and (5.5). Such an integral solution is called a virtual trajectory $C$ of the physical system $S_{k}$. The complete physical system $S_{k}$ is composed of actual and virtual trajectories $C$. Thus the complete physical system $S_{k}$ consists of all the integral solutions of the intrinsic differential equations (5.8).

The virtual trajectories $C$ of a physical system $S_{k}$ of a field of force are the actual trajectories $C$ of the physical system $S_{k}$ of the reversed field of force $-\Phi$, for which the original force vector $\Phi$ is multiplied by the scalar $(-1)$. 
6. The differential equations of a velocity system $S_{\infty}$. A velocity system $S_{\infty}$ of $\infty^{2 n-1}$ curves in $V_{n}$, is defined by the equations

$$
m \dot{v}=m v \frac{d v}{d s}=T_{1}=g_{\alpha \beta} \Phi^{x} \frac{d x^{\beta}}{d s}=\Phi_{\alpha} \frac{d x^{\alpha}}{d s}
$$

$$
m v_{0}^{2}=r N, \quad \Phi^{i}=T_{1} \frac{d x^{i}}{d s}+r N x^{i}
$$

Theorem. 6.1. - A velocity system $S_{\infty}$ of $\infty^{2 n-1}$ curves $O$ in Riemannian space $V_{n}$, is given by the equations

$$
m v \frac{d v}{d s}=g_{\alpha \beta} \Phi^{\alpha} \frac{d x^{\beta}}{d s}, \quad m v_{0}^{2}=r N
$$

$$
r N x^{i}=\Phi^{i}-\left(g_{\alpha \beta} \Phi^{\alpha} \frac{d x^{\beta}}{d s}\right) \frac{d x^{i}}{d s}
$$

for $i=1,2, \ldots, n$.

For, the two systems of equations (6.1) and (6.2) are equivalent.

THEOREM 6.2. - Either one the two systems of $n$ ordinary second order differential equalions

$$
\begin{aligned}
& m v_{0}^{2} a^{i}=\left(g_{\alpha \beta} \dot{x^{\alpha}} \dot{x}^{\beta}\right) \Phi^{i}+\frac{\left(g_{\alpha \beta} \Phi^{\alpha} x^{\beta}\right)}{\left(g_{\alpha \beta} \dot{x^{\alpha}} \dot{x^{\beta}}\right)}\left(v_{0}^{2}-g_{\alpha \beta} \dot{x^{\alpha}} \dot{x}^{\beta}\right) \dot{x}^{i} \\
& m v_{0}^{2} \alpha_{i}=\left(g_{\alpha \beta} \dot{x^{\alpha}} \dot{x}^{\beta}\right) \Phi_{i}+\frac{\left(g_{\alpha \beta} \Phi^{\alpha} \dot{x}^{\beta}\right)}{\left(g_{\alpha \beta} \dot{x} \dot{x}^{\beta}\right)}\left(v_{0}^{2}-g_{\alpha \beta} \dot{x}^{\alpha} \dot{x}^{\beta}\right)\left(g_{i \alpha^{\alpha}} \dot{x}^{\alpha}\right) ;
\end{aligned}
$$

for $i=1,2, \ldots, n$, defines a velocity system $S_{\infty}$ of $\infty^{2 n-1}$ curves $C$ in Rieman. nian space $V_{n}$.

For, by $(2.9)$ and $(6.2)$, it is seen that

$$
m v_{0}^{2} \alpha^{i}=v^{2} \Phi^{i}+\frac{\left(g_{\alpha \beta} \Phi^{\alpha} \dot{x}^{\beta}\right)}{v^{2}}\left(v_{0}^{2}-v^{2}\right) \dot{x^{i}}
$$

Since $v^{2}=g_{\alpha \beta} \dot{x^{\alpha}} \dot{x}^{\beta}$, the first set of equations $(6.3)$ is obtained.

The first set of equations (6.3), is the contravariant form of a velocity system $S_{\infty}$. The second set of equations (6.3), is the covariant form of a velocity system $S_{\infty}$. 
J. De Crcco: The Riemannian Geometry of Physical Systems, etc. 351

Theonem 6.3. - The equations of Lagrange for a velocity system $S_{\infty}$, in a Riemannian space $V_{n}$, are

$$
r N\left[\frac{d}{d t}\left(\frac{\partial T}{\partial \dot{x}^{i}}\right)-\frac{\partial T}{\partial x^{i}}\right]-2 T \Phi_{i}-\frac{\left(g_{\alpha \beta} \Phi^{x} \dot{x}^{\beta}\right)}{2 T^{\prime}}(r N-2 T) \frac{\partial T}{\partial \dot{x}^{i}}=0,
$$

for $i=1,2, \ldots, n$.

This follows equations (2.5), (2.6), and (6.3).

THEOREM 6.4. - The intrinsinc differential equations of the $\infty^{2 n-1}$ curves $C$ of a velocity system $S_{\infty}$, of a Riemannian space $\nabla_{n}$, are

$$
N \frac{d r}{d s}=T_{1}-r N_{2}, r \frac{d N}{d s}=-T_{1}+r N_{2}, r N x^{i}=\Phi^{i}-T_{1} \frac{d x^{i}}{d s}
$$

for $i=1,2, \ldots, n$. A velocity system $S_{\infty}$, is the limiting case of a physical system $S_{k}$ as $k$ becomes infinite.

The equations (6.6) are obtained by eliminating the constant speed $v_{0}>0$, from the equations (6.2) and using the equations (3.12).

As $k$ becomes infinite, the limiting set of the intrinsic differential equations (5.8) of a physical system $S_{k}$, is the set of intrinsinc differential equa. tions $(6.6)$ of a velocity syistem $S_{\infty}$. Therefore a velocity system $S_{\infty}$, is the limiting case of a physical system $S_{k}$ as $k$ becomes infinite.

The three systems of differential equations (6.2), (6.3), and (6.5), are equivalent. Any integral solution of any one of these three equivalent systems, is called an actual velocity curve C. An integral solution of the intrinsic differential equations $(6.6)$, which does not satisfy any one of the three equivalent systems (6.2), (6.3), and (6.5), is called a virtual velocity curve $C$. The complete velocity system $S_{\infty}$, is composed of the actual and virtual velocity curves $C$. Therefore the complete velocity system $S_{\infty}$ consists of all the integral solutions of the intrinsic differential equations (6.6).

The virtual velocity curves $C$ of a velocity system $S_{\infty}$ of a field of force $\Phi$, are actual velocity curves $C$ of the velocity system $S_{\infty}$ of the field of force $-\Phi$, for which the original force vector $\Phi$ is multiplied by the sca$\operatorname{lar}(-1)$.

7. The explicit form of the differential equations of a complete physical system $S_{k}$ where $k \neq-1$. Let $j$ denote a fixed index. The symbol $\Delta^{i}$ for $i=1,2, \ldots, n$, denotes the expression

$$
\Delta^{i}=\frac{d^{2} x^{i}}{d\left(x^{j}\right)^{2}}+\Gamma_{\alpha \beta}^{i} \frac{d x^{\alpha}}{d x^{j}} \frac{d x^{\beta}}{d x^{j}}-\Gamma_{\alpha \beta}^{j} \frac{d x^{\alpha}}{d x^{j}} \frac{d x^{\beta}}{d x^{j}} \frac{d x^{i}}{d x^{j}} .
$$


Evidently $\Delta^{j}=0$. The expanded form of this, is

$$
\begin{aligned}
& \Delta^{i}=\frac{d^{2} x^{i}}{d\left(x^{j}\right)^{2}}+\Gamma_{j j}^{i}+\left(2 \Gamma_{i j}^{i}-\Gamma_{j j}^{j}\right) \frac{d x^{i}}{d x^{j}}+2 \underset{\alpha \neq i, j}{\Sigma} \Gamma_{\alpha j}^{i} \frac{d x^{\alpha}}{d x^{j}} \\
& +\left(\Gamma_{i i}^{i}-2 \Gamma_{i j}^{j}\right) \frac{d x^{i}}{d x^{j}} \frac{d x^{i}}{d x^{j}}+2 \underset{\alpha \neq i, j}{\Sigma}\left(\Gamma_{\alpha i}^{i}-\Gamma_{\alpha j}^{j}\right) \frac{d x^{\alpha}}{d x^{j}} \frac{d x^{i}}{d x^{j}} \\
& \quad-\Gamma_{i i}^{j} \frac{d x^{i}}{d x^{j}} \frac{d x^{i}}{d x^{j}} \frac{d x^{i}}{d x^{j}}-2 \underset{\alpha \neq i, j}{\Sigma} \Gamma_{\alpha i}^{j} \frac{d x^{\alpha}}{d x^{j}} \frac{d x^{i}}{d x^{j}} \frac{d x^{i}}{d x^{j}} \\
& -\sum_{\substack{\alpha \neq i, j \\
\beta \neq i, j}}^{\Sigma} \Gamma_{\alpha \beta}^{j} \frac{d x^{\alpha}}{d x^{j}} \frac{d x^{\beta}}{d x^{j}} \frac{d x^{i}}{d x^{j}} .
\end{aligned}
$$

The vector geodesic curvature $x$ of a curve $C$ in $V_{n}$, is given in terms of the symbol $\Delta^{i}$ by the equations

$$
x^{i}=\frac{d x^{i}}{d x^{j}} x^{i}+\Delta^{i}\left(\frac{d x^{j}}{d s}\right)^{2}
$$

where $j$ is fixed and $i=1,2, \ldots, n$.

The vector geodesic curvature $x$ of a carve $C$ in $\nabla_{n}$, is parallel to the unit tangent vector $\tau_{1}=\frac{d x}{d s}$, if and only if $x$ is the zero vector. Therefere since the conditions for a geodesic $C$ of $\nabla_{n}$, are $x^{i}=0$, for $i=1,2, \ldots, n$, it follows that a curve $C$, of $\nabla_{n}$, is a geodesic of $V_{n}$ if and only if

$$
\Delta^{i}=0
$$

for $i=1,2, \ldots, n$.

For a physical system $S_{k}$ where $k \neq-1, \infty$, it is found by $(5.2)$ and (7.3), that

$$
m\left(\frac{d x^{j}}{d t}\right)^{2} \Delta^{i}=(1+k)\left(\Phi^{i}-\Phi^{j} \frac{d x^{i}}{d x^{j}}\right)
$$

where $j$ is fixed, and $i=1,2, \ldots, n$. 
Therefore for a physical system $S_{k}$ where $k \neq-1$, we must have

$$
\frac{\Delta^{l}}{\Delta^{i}}=\frac{\Phi^{l}-\Phi^{j} \frac{d x^{l}}{d x^{j}}}{\Phi^{i}-\Phi^{j} \frac{d x^{i}}{d x^{j}}}
$$

where $j$ is fixed, and $i, l=1,2, \ldots, \mathrm{n}$. These equations are true for a velocity system $S_{\infty}$ since it is the limiting case of a physical system $S_{k}$ as $h$ approaches infinity.

Differentiate the equation (5.5) with respect to $x^{i}$. The answer can be written in the form

$$
\left(\Phi^{i}-\Phi^{j} \frac{d x^{i}}{d x^{j}}\right) \frac{d \Delta^{i}}{d x^{j}}=-\Phi^{j} \Delta^{i} J^{i}-\frac{2 \frac{d^{2} x^{j}}{d t^{2}}}{\left(\frac{d x^{j}}{d t}\right)^{2}}\left(\Phi^{i}-\Phi^{j} \frac{d x^{i}}{d x^{j}}\right) \Delta^{i}
$$

$$
+\left[\frac{d \Phi^{i}}{d x^{j}}-\frac{d \Phi^{j}}{d x^{j}} \frac{d x^{i}}{d x^{j}}+\Phi^{j}\left(\Gamma_{\alpha \beta}^{i} \frac{d x^{\alpha}}{d x^{j}} \frac{d x^{\beta}}{d x^{j}}-\Gamma_{\alpha \beta}^{j} \frac{d x^{\alpha}}{d x^{j}} \frac{d x^{\beta}}{d x^{j}} \frac{d x^{i}}{d x^{j}}\right)\right] \Delta^{i}
$$

By (5.3) and (7.5), it is found that

$$
\begin{gathered}
\frac{-2 \frac{d^{2} x^{j}}{d t^{2}}}{\left(\frac{d x^{j}}{d t}\right)^{2}}\left(\Phi^{i}-\Phi^{j} \frac{d x^{i}}{d x^{j}}\right) \Delta^{i}=\left[-2 \Phi^{j}+\frac{2 k\left(g_{\alpha \beta} \Phi^{\alpha} \frac{d x^{\beta}}{d x^{j}}\right)}{(1+k)\left(g_{\alpha \beta} \frac{d x^{\alpha}}{d x^{j}} \frac{d x^{\beta}}{d x^{j}}\right)}\right] \Delta^{i} \Delta^{i} \\
+2 \Gamma_{\alpha \beta}^{j} \frac{d x^{\alpha}}{d x^{j}} \frac{d x^{\beta}}{d x^{j}}\left(\Phi^{i}-\Phi^{j} \frac{d x^{i}}{d x^{j}}\right) \Delta^{i}
\end{gathered}
$$

Theonem 7.1 - The explicit differential equations of a complete physical system $S_{k}$ where $k \neq-1$, in a Riemannian space $V_{n}$, are

$$
\frac{\Delta^{l}}{\Delta^{i}}=\frac{\Phi^{l}-\Phi^{j} \frac{d x^{l}}{d x^{j}}}{\Phi^{i}-\Phi^{j} \frac{d x^{i}}{d x^{j}}}
$$




$$
\left(\Phi^{i}-\Phi^{j} \frac{d x^{i}}{d x^{j}}\right) \frac{d \Delta^{i}}{d x^{j}}=\left[-3 \Phi^{j}+\frac{2 k\left(g_{\alpha \beta} \Phi^{\alpha} \frac{d x^{\beta}}{d x^{j}}\right)}{(1+k)\left(g_{\alpha \beta} \frac{d x^{x}}{d x^{j}} \frac{d x^{\beta}}{d x^{j}}\right)}\right] \Delta^{i} \Delta^{i}
$$

$$
+\left[\begin{array}{c}
\frac{d \Phi^{i}}{d x^{j}}-\frac{d \Phi^{j}}{d x^{j}} \frac{d x^{i}}{d x^{j}}+\Phi^{j}\left(\Gamma_{\alpha \beta}^{i} \frac{d x^{\alpha}}{d x^{j}} \frac{d x^{\beta}}{d x^{j}}-\Gamma_{\alpha \beta}^{j} \frac{d x^{\alpha}}{d x^{j}} \frac{d x^{\beta}}{d x^{j}} \frac{d x^{i}}{d x^{j}}\right) \\
+2 \Gamma_{\alpha \beta}^{j} \frac{d x^{\alpha}}{d x^{j}} \frac{d x^{\beta}}{d x^{j}}\left(\Phi^{i}-\Phi^{j} \frac{d x^{i}}{d x^{j}}\right)
\end{array}\right] \Delta^{i},
$$

where the two indices $i$ and $j$ with $i \neq j$ are held fixed, and $l \neq i, j$, is allowed to vary over the remaining integers 1 to $n$. This is a system of $(n-1)$ ordinary differential equalions in which there are $(n-2)$ ordinary second order differential equations and one ordinary third order differential equation. As $k$ becomes infinite, the limiting set of 17.91 , is composed of the explicit differential equations of a complete velocity system $\mathbb{S}_{50}$.

For, the system of ordinary differential equations (7.9), is found by means of equations (7.6), (7.7), and (7.8). By Theorem 6.4 the limiting set of (7.9), is composed of the explicit differential equations of a complete velocity system $S_{\infty}$.

From (7.9), the number of constants of integration is $(n-2) 2+3=$ $=2 n-4+3=2 n-1$. Therefore, a complete physical system $S_{k}$ where $k \neq-1$, consists of $\infty^{2 n-1}$ trajectories $C$.

By equations (7.9), it is clear the $\infty^{2 n-1}$ geodesics $C$ of the Riemannian space $V_{n}$, belong to every complete physical system $S_{k}$ where $k \neq-1$. These are the trajectories $C$ of the complete physical system $S_{k}$, which correspond to infinite initial speed.

A system of ordinary differential equations in Riemannian space $V_{n}$, is said to be of the type $(G)$, if and only if it can be written in the form

$$
\begin{aligned}
& \frac{\Delta^{l}}{\Delta^{i}}=\frac{\Phi^{l}-\Phi^{j} \frac{d x^{l}}{d x^{j}}}{\Phi^{i}-\Phi^{j} \frac{d x^{i}}{d x^{j}}}, \\
& \frac{d \Delta^{i}}{d x^{j}}=G^{i} \Delta^{i}+H^{i} \Delta^{i} \Delta^{i},
\end{aligned}
$$

where the two indices $i$ and $j$ with $i \neq j$, are held fixed, and $l \neq i$, is allowed to vary over the remaining integers $1,2, \ldots, n$. For a fixed $i$, 
$G^{i}$ and $H^{i}$, are two single valued functions of at least class one in a $(2 n-1)$ dimensional region of $R$ of lineal elements $E\left(x^{1}, x^{2}, \ldots, x^{n} ; \frac{d x^{1}}{d x^{j}}, \frac{d x^{2}}{d x^{j}}, \ldots\right.$, $\left.\frac{d x^{i-1}}{d x^{j}}, \frac{d x^{j+1}}{d x^{j}}, \ldots, \frac{d x^{n}}{d x^{j}}\right)$. For $i=1,2, \ldots, n$, the $\Phi^{i}$ form a contravariant vector function of at least class two in this same region $R$ of lineal elements $E$, such that the contravariant vector $\Phi^{i}$ is not the zero vector, and its direction is not identical with that of the corresponding lineal element $E$, for all lineal elements $E$ in this region $R$.

The collection of all curves $C$ which are integral solutions of the system of ordinary differential equations $(7.10$, is said to be a system of the type $(G)$ [6]. A system of the type $(G)$, possess $\infty^{2 n-1}$ curves $C$.

The totality of $\infty^{2 n-2}$ geodesies of the Riemannian space $V_{n}$, is part of every system of the type $(G)$.

Every system of $\infty^{2 n-1}$ curves $O$ of the type $(G)$, possesses the Property $\mathrm{I}$. This may be described in the following manner. For a given lineal element $E$ of the region $R$, there are $\infty^{1}$ curves $C$ of the system of the type $(G)$, which pass through the point $x$ of $E$, in the direction of $E$. Depending on this lineal element $E$, there is determined one and only direction $\tau$, which is the direction $\tau$ of the non-zero contravariant vector $\Phi^{i}$, such that this direction $\tau$ is in each osculating geodesic surface at the point $x$ of the lineal element $E$, of every one of the curves $C$ of the system of the type $(G)$, which passes through the point $x$ of $E$, in the direrection of $E$. This direction $\tau$ does not coincide with that of $E$.

Upon comparing the equations (7.9) and (7.10), it follows that every complete physical system $S_{k}$ where $k \neq-1$, is of the type $(G)$. However, not every system of type $(G)$, is a complete physical system $S_{k}$ where $k \neq-1$.

A physical system $S_{k}$ where $k \neq-1$, possesses the stronger form of Property I. This signifies that the direction $\tau$ of Property $I$, is a function of the position $x$ only.

Theorem 7.2 - Consider a fixed $k \neq-1$, which may be infinite, such that $k \neq-3$ if the dimension $n$ of the Riemannian space $V_{n}$, is two. Two arbitrary positional fields of force $\bar{\Phi}$ and $\Phi$, possess the same complete physical system $S_{k}$, if and only if the force vector $\bar{\Phi}$, is a non-zero constant scalar multiple $c$ of the force vector $\Phi$. Thus $\bar{\Phi}=c \Phi$, where $c \neq 0$, is a constant scalar.

Essentially this proposition states that there are only $\infty^{1}$ positional fields of force $\Phi$, which possess the same complete physical system $S_{k}$ where $k \neq-1$, and $k \neq-3$, if $n=2$.

If $c>0$, the actual and virtual trajectories of the complete physical system $S_{k}$ of the positional field of force $\Phi$, correspond respectively, to the 
actual and virtual trajectories of the same complete physical system $S_{k}$ of the positional field of force $\bar{\Phi}=c \Phi$, On the other hand, if $c<0$, the actual and virtual trajectories of the complete physical system $S_{k}$ of the positional field of force $\Phi$, correspond respectively, to the virtual and actual trajectories of the same complete physical system $S_{k}$ of the positional field of force $\bar{\Phi}=c \Phi$. Thus when $c<0$, the actual and virtual trajectories are interchanged.

The above proposition is not true when $n=2$, and $k=-3$. This is demonstrated by the following example. In the Euclidean plane, let $(x, y)$ denote rectangular coordinate of a point $P$. The totality of $\infty^{3}$ circles and $\infty^{2}$ straight lines in the Euclidean plane, is a complete physical system $S_{3}$. The rectangular components of any positional field of force which possesses this complete physical system $S_{-3}$, are $\Phi^{1}=a x+h, \Phi^{2}=a y+k$, where $a, h, k$, are three constants, not all zero. According as $a \neq 0$, or $a=0$, the positional field of force $\Phi$, is elastic or Galilean. Therefore, there are $\infty^{3}$ positional fields of force $\Phi$ in the Euclidean plane, which possess the same complete physical system $S_{-3}$, composed of the $\infty^{3}$ circles and the $\infty^{2}$ straight lines. Consequently when $n=2$, and $k=-3$, Theorem 7.2 , is not true.

The proof of Theorem 7.2, is as follows.

The condition $\bar{\Phi}=c \Phi$, where $\mathbf{c} \neq 0$, is a constant scalar, is a sufficient condition even in the case when $n=2$, and $k=-3$. This is demonstrated by substituting $\bar{\Phi}=c \Phi$, into the equations (7.9).

The major part of the proof, is to show that $\bar{\Phi}=c \Phi$, where $c \neq 0$, is a constant scalar, is a necessary condition provided that $k \neq-1$, and $k \neq-3$, if $n=2$.

Accordingly, let $\Phi$ and $\bar{\Phi}$ denote two arbitrary positional fields of force which possess the same complete physical system $S_{k}$ where $k \neq-1$, and $k \neq-3$, if $\mathrm{n}=2$.

It will be proved that a necessary condition for this, is that there exist a non-zero scalar function $\rho=\rho(x)$, of class two in the given region of points $x$, such that

$$
\bar{\Phi}^{i}=\rho \Phi^{i}
$$

for $i=1,2, \ldots, n$.

Let $n \geqq 3$. By the first (n-2) equations of (7.9), a necessary condition for the validity of the proposition, is that the following set of $(n-2)$ identities hold, namely

$$
\frac{\bar{\Phi}^{l}-\bar{\Phi}^{j} \frac{d x^{l}}{d x^{j}}}{\bar{\Phi}^{\imath}-\bar{\Phi}^{j} \frac{d x^{i}}{d x^{j}}}=\frac{\Phi^{l}-\Phi^{j} \frac{d x^{l}}{d x^{j}}}{\Phi^{i}-\Phi^{j} \frac{d x^{i}}{d x^{j}}},
$$


where $i$ and $j$ with $i \neq j$, are fixed, and $l \neq i, j$, varies over the remaining integers $1,2, \ldots, n$.

Set $\frac{d x^{i}}{d x^{j}}=0$, in (7.12). From the resulting identities, it is found that

$$
\frac{\bar{\Phi}^{l}}{\Phi^{i}}=\frac{\bar{\Phi}^{i}}{\Phi^{i}}=\frac{\bar{\Phi}^{j}}{\Phi^{j}}=\rho \neq 0
$$

where $i$ and $j$. with $i \neq j$, are fixed, and $l \neq i, j$, varies over the remaining integers 1, 2, ..., n. From these, are deduced the equations (7.11). By (7.11), the identities (7.12), are valid.

Consequently the necessary set of conditions (7.11), have been established when $n \geq 3$.

From (7.9), another necessary condition for the validity of the propo. sition, is

$$
\begin{aligned}
& \left(\Phi^{i} \frac{d x^{j}}{d s}-\Phi^{j} \frac{d x^{i}}{d s}\right)\left[-3 \bar{\Phi}^{j}+\left(\frac{2 k}{1+k}\right)\left(\bar{\Phi}_{\alpha} \frac{d x^{x}}{d s}\right) \frac{d x^{j}}{d s}\right] \\
= & \left(\bar{\Phi}^{i} \frac{d x^{j}}{d s}-\bar{\Phi}^{j} \frac{d x^{i}}{d s}\right)\left[-3 \Phi^{j}+\left(\frac{2 k}{1+k}\right)\left(\Phi_{\alpha} \frac{d x^{x}}{d s}\right) \frac{d x^{j}}{d s}\right],
\end{aligned}
$$

where $i$ and $j$ with $i \neq j$, are fixed.

When $n=2$, the indices $i$ and $j$ can be taken as $i=l$ and $j=2$ The preceding condition ban be written as

$$
\begin{gathered}
3\left(\Phi^{1} \bar{\Phi}^{2}-\bar{\Phi}^{1} \Phi^{2}\right)-\left(\frac{2 k}{1+k}\right)\left(\bar{\Phi}_{1} \frac{d x^{1}}{d s}+\bar{\Phi}_{2} \frac{d x^{2}}{d s}\right)\left(\Phi^{1} \frac{d x^{2}}{d s}-\Phi^{2} \frac{d x^{1}}{d s}\right) \\
+\left(\frac{-2 k}{1+k}\right)\left(\Phi_{1} \frac{d x^{1}}{d s}+\Phi_{2} \frac{d x^{2}}{d s}\right)\left(\bar{\Phi}^{1} \frac{d x^{2}}{d s}-\bar{\Phi}^{2} \frac{d x^{1}}{d s}\right)=0
\end{gathered}
$$

From this identity, the following three conditions are found, namely

$$
\left(\frac{3+k}{1+k}\right)\left(\Phi^{1} \bar{\Phi}^{2}-\bar{\Phi}^{1} \Phi^{2}\right) g_{i j}=0
$$

where $i, j=1,2$.

Since $g=g_{11} g_{22}-g_{12}^{2}>0$, and since $k \neq-1,-3$, it follows from (7.16), that the necessary set of conditions (7.11), must be valid even when $\mathrm{n}=2$, provided that $k \neq-1,-3$. 
Therefore, under all possible circomstances, the necessary set of conditions (7.11), has been derived.

With the aid of (7.11), it is seen by (7.9), that the final necessary condition for the validity of the proposition, is

$$
\frac{1}{\rho}\left[\frac{d}{d x^{j}}\left(\rho \Phi^{i}\right)-\frac{d}{d x^{j}}\left(\rho \Phi^{j}\right) \frac{d x^{i}}{d x^{j}}\right]=\frac{d \Phi^{i}}{d x^{j}}-\frac{d \Phi^{j}}{d x^{j}} \frac{d x^{i}}{d x^{j}},
$$

where $i$ and $j$ with $i \neq j$, are fixed. Then

$$
\frac{d \rho}{d x^{j}}\left(\Phi^{i}-\Phi^{i} \frac{d x^{i}}{d x^{i}}\right)=0
$$

Since $i$ and $j$ with $i \neq j$, can be so selected that $\left(\Phi^{i}-\Phi^{j} \frac{d x^{i}}{d x^{j}}\right) \neq 0$, it follows that $\frac{d \rho}{d x^{j}}=0$. This means that $\rho=c \neq 0$, is constant.

Therefore under the given bypotheses, the sufficient condition that $\bar{\Phi}=c \Phi$, where $c \neq 0$, is a constant scalar, has been proved to be a necessary condition. Consequently the proof of Theorem 7.2, is complete.

8. Kasner's theorem concerning the initial curvatures of the rest trajectories of a complete physical system $S_{k}$ where $k \neq-1,-3 .[7] . A$ rest trajectory $C$ of a complete physical system $S_{k}$ where $k \neq-1$, but where $k$ may be infinite, is a trajectory $C$ of the given $S_{k}$, which starts from a given initial point $x_{0}$ with velocity vector equal to the zero vector. In a given complete physical system $S_{k}$ where $k \neq-1$, of $\infty^{2 n-1}$ trajectories, there are $\infty^{n}$ rest trajecories, under certain general conditions.

In this section, a proof of a theorem of KASNER concerning the initial curvature of a rest trajectory of a complete physical system $S_{k}$ where $k \neq-1,-3$ but where $k$ may be infinite, in a Riemannian space $V_{n}$, will be given. This result has been proved only for the eases of a Euclidean plane, a Euclides $n$ space of three dimensions, and a surface.

By (3.8), (3.12), and (5.8), the intrinsic differential equations of a complete physical system $S_{k}$ where $k \neq-1$, ean be written in form

$$
\begin{gathered}
\frac{d T_{1}}{d s}=T_{2}+\frac{N}{r}, \frac{d N}{d s}=N_{2}-\frac{T_{1}}{r} \\
N \frac{d r}{d s}=\left(\frac{3+k}{1+k}\right) T_{1}-r N_{2}, \quad N \tau_{2}^{i}=\Phi^{i}-T_{1}^{T} \tau_{1}^{i} .
\end{gathered}
$$


From (3.9) and the above equations, it follows that

$$
\begin{aligned}
& \Phi^{i}=T_{1} \tau_{1}^{i}+N \tau_{2}^{i}, \\
& \quad \frac{D \Phi}{d s}=T_{2} \tau_{1}^{i}+N_{2} \tau_{2}^{i}+\frac{N}{r_{2}} \tau_{3}^{i},
\end{aligned}
$$

where $r_{2}$ is the second radius of curvature of a trajectory $C$ of the complete physical system $S_{k}$ for $k \neq-1$.

THeorem 8.1 - In a given complete physical system $S_{k}$ where $k \neq-1$, there are $\infty^{1}$ trajectories $C$ which contain a given lineal element $E$. By varving $k$, there are $\infty^{2}$ such trajectories $C$. All these $\infty^{2}$ trajectories $C$ which contain the given lineal element $E$, have the same osculating geodesic surface at E. Moreover, at this lineal element E, they possess the same second curvature $\left|\boldsymbol{x}_{2}\right|=1 / r_{2}$, given by the formula

$$
N\left|x_{2}\right|=\frac{N}{r_{2}}=\tau_{3} \cdot \frac{D \Phi}{d s} .
$$

This result is a consequence of the equations (8.1) and (8.2).

THeOReM 8.2 - The rest trajectory $C$ of any complete physical system $S_{\boldsymbol{h}}$ where $k \neq-1$, which passes through a fixed point $x$, is tangent to the line of force at this point $x$. Also the space derivative $\frac{D \Phi}{d s}$, of the force vector $\Phi$, relative to this rest trajectory $C$, evaluated at the initial point $x$, is tangent to the osculating geodesic surface of the rest trajectory $C$, at this point $x$.

For, since $C$ is the rest trajectory of the complete physical system $S_{k}$ where $k \neq-1$, at the given point $x$, then $\dot{x}^{i}=\frac{d x^{i}}{d t}=0$, for a particle on the rest trajectory $C$ at the point $x$. From the equations

$$
m\left(\frac{d x^{i}}{d t}\right)^{2} \Delta^{i}=(1+k)\left(\Phi^{2}-\Phi^{j} \frac{d x^{i}}{d x^{j}}\right)
$$

$$
\frac{\Delta^{\prime}}{\bar{\Delta}^{i}}=\frac{\Phi^{l}-\Phi^{j} \frac{d x^{l}}{d x^{j}}}{\Phi^{i}-\Phi^{j} \frac{d x^{i}}{d x^{j}}},
$$

where $i$ and $j$ with $i \neq j$, are fixed, and $l \neq i, j$, varies over the remaining integers $1,2, \ldots, n$, it follows that the direction of the rest trajectory $C$ 
at this point $x$, is given by

$$
\frac{d x^{\alpha}}{d x^{j}}=\frac{\Phi^{x}}{\Phi^{i}}
$$

where $j$ is fixed, and $\alpha \neq j$, varies over the remaining integers $1,2, \ldots, n$. Of course, $j$ is chosen so that $\Phi^{j} \neq 0$. Therefore, the rest trajectory $C$ is tangent to the line of force at the initial point $x$.

From the proof of the proceding paragraph, it follows that at the initial point $x$ of a rest trajectory $C$, the following relations must hold, namely

$$
T_{1}=|\Phi| \neq 0 \text {, and } N=0 \text {. }
$$

By means of (8.2) and (8.6), the space derisative $\frac{D \Phi}{d s}$, of the force vee. tor $\Phi$, relative to the rest trajectory $C$, evaluated at the initial point $x$ of $C$, is tangent to the geodesic surface of this trajectory $C$ at its initial point $x$.

THeоRem 8.2 - The Theorem of Kasner Concerning The Initial Curvature of a Rest Trajectory, Let the curvature $\left|x_{0}\right|=1 / r_{0}$, of the line of force at a given point $x$, be not zero. The rest trajectory $C$ of the complete physical system $S_{k}$ where $k \neq-1,-3$, with this point $x$ as its initial point, is tangent to the line of force at this point $x$. The curvature $|x|=1 / r$, of this rest trajectory $C$, is not zero at its initial point $x$. The ratio $\rho=|x| /\left|x_{0}\right|=r_{0} \mid r$, of the curvature $|x|=1 / r$, of the rest trajectory $C$, and of the curvature $\left|x_{0}\right|=1 / r_{0}$, of the line of force, at the initial point $x$ of the rest trajectory $C$, is

$$
\rho=\frac{|x|}{\left|x_{0}\right|}=\frac{r_{0}}{r}=\frac{1+k}{3+k}
$$

At the initial point $x$, the rest velocity curve $C$ and the line of force, possess the same curvature.

For, under the given hypothesis, $r_{0} \neq 0$, and $T_{1}=|\Phi| \neq 0, N_{2} \neq 0$, at the initial point $x . B y(3.18)$, we have $T_{1}=r_{0} N_{2}$. By (8.1), and (8.6), it is seen that

$$
\rho=\frac{|x|}{\left|x_{0}\right|}=\frac{r_{0}}{r}=\frac{r_{0} N_{2}}{T_{1}}\left(\frac{1+k}{3+k}\right)=\frac{1+k}{3+k} \text {. }
$$

This completes the proof of the above theorem of KASNER.

For dynamical trajectories, this ratio $\rho$ is $1 / 3$. For general catenaries, this $\rho$ is $1 / 2$. For general brachistochrones, this ratio $\rho$ is -1 . For velocity curves, this ratio $p$ is 1 . 
Elsewhere this ratio has been studied for directional fields of force in which the force vector at any point depends not only in the position of the point but also on the direction passing through the point [8].

9. Curvature trajectories and velocity families. A family $\Omega$ of $\infty^{2 n-2}$ curves $\omega$ in a Riemannian space $V_{n}$, which is not the geodesic family, can be defined as the $\infty^{2 n-2}$ integral curves $\omega$ of a system of $n$ ordinary second order differential equations of the form

$$
x^{i}=\Phi^{i}
$$

for $1,2, \ldots, n$, where the $\Phi^{i}$ for $i=1,2, \ldots, n$, form a contravariant vector function of at least class two in a $(2 n-1)$ dimensional region $R$ of lineal elements $E\left(x^{1}, x^{2}, \ldots, x^{n} ; \frac{d x^{1}}{d x^{j}}, \frac{d x^{2}}{d x^{j}}, \ldots, \frac{d x^{j-1}}{d x^{j}}, \frac{d x^{j+1}}{d x^{j}}, \ldots, \frac{d x^{n}}{d x^{j}}\right)$, such that the contravariant vector $\Phi^{i}$ is not the zero vector, and its direction is not identical with that of the corresponding lineal element $E$, for all lineal elements $E$ of this region $R$. The $x^{i}$ in these equations, are the contravariant form of the vector geodesic curvature $x$ of any curve $\omega$ of this family $\Omega$.

Sometimes it is found convenient to think of the $\Phi^{i}$ as being functions of $(x ; \dot{x})=\left(x^{1}, x^{2}, \ldots, x^{n} ; \dot{x}^{1}, \dot{x}^{2}, \ldots, \dot{x}^{n}\right)$, where $\dot{x}^{i}=\frac{d x^{i}}{d t}$, for $i=1,2, \ldots, n$. Then for each $i$, the function $\Phi^{i}$ is homogeneous of degree zero in the $\dot{x}$.

A curvature trajectory $C$ of the family $Q$ of $\infty^{2 n-2}$ carves $\omega$, is defined in the following manner [9]. A geodesic $C$ of the Riemannian space $V_{n}$, is regarded to be a curvature trajectory $C$ of this family $\Omega$. Henceforth, let $C$ be a curve such that its vector geodesic curvature $x$ is not zero at every one of its points $x$. To this curve $C$, there is associated a constant scalar $c \neq 0$. Such a curve $C$ is said to be a curvature trajectory $C$ of the family $\mathbf{\Omega}$ if when the unique curve $\omega$ of the family $Q$, is constructed such that $\omega$ passes through any given point $x$ of the curve $C$ in the direction of this curve $C$, the two curves $C$ and $\omega$ possess the same osculating geodesic surface at this point $x$, and the curvature $|x|=1 / r$, of $C$, is a constant multiple $c \neq 0$, of the curvature $\left|x_{0}\right|=1 / r_{0}$, of $\omega$, at this point $x$.

The system $\Gamma$ of $\infty^{2 n-2}$ curvature trajectures $C$ of the family $Q$ of $\infty^{2 n-2}$ curves $\omega$, is composed of the $\infty^{2 n-1}$ integral curves $C$ of the system of $n$ ordinary second order differential equations.

$$
x^{i}=c \Phi^{i},
$$

for $i=1,2, \ldots, n$, where $c$ is an arbitrary constant of integration. If $c=0$, these define the geodesic curvature trajectories of the family $\mathbf{Q}$. Henceforth, its is supposed that $c \neq 0$. 
By (7.3), the system of equations (9.2), is found to be equivalent to the system of $(n-1)$ equations

$$
\left(\frac{d x^{i}}{d s}\right)^{2} \Delta^{i}=c\left(\Phi^{i}-\Phi^{j} \frac{d x^{i}}{d x^{i}}\right)
$$

where $j$ is fixed, and $i \neq j$, varies over the remaining integers $1,2, \ldots, n$.

From $(9.3)$, is dedueed the set of $(n-2)$ equations

$$
\frac{\Delta^{l}}{\Delta^{i}}=\frac{\Phi^{l}-\Phi^{j} \frac{d x^{l}}{d x^{i}}}{\Phi^{i}-\Phi^{j} \frac{d x^{i}}{d x^{i}}}
$$

where $i$ and $j$ with $i \neq j$, are fixed, and $l \neq i, j$, varies over the remaining integers $1,2, \ldots, n$.

Theonem $9.1-A$ system $\Gamma$ of $\infty^{2 n-1}$ curvature trajectories $O$ of a family $\Omega$ of $\infty^{2 n-2}$ curves $\omega$, is given by the set of $(n-1)$ ordinary differential equations

$$
\frac{\Delta^{l}}{\Delta^{i}}=\frac{\Phi^{l}-\Phi^{i} \frac{d x^{l}}{d x^{j}}}{\Phi^{i}-\Phi^{j} \frac{d x^{i}}{d x^{j}}}
$$

(9.5) $\quad\left(\Phi^{i}-\Phi^{i} \frac{d x^{i}}{d x^{i}}\right) \frac{d \Delta^{i}}{d x^{j}}=\left[-3 \Phi^{j}+\left(\frac{\partial \Phi^{i}}{\partial x^{\gamma}}-\frac{\partial \Phi^{j}}{\partial x^{\gamma}} \frac{d x^{i}}{d x^{j}}\right)\left(\frac{\Phi^{\gamma}-\Phi^{j} \frac{d x^{\gamma}}{d x^{j}}}{\Phi^{i}-\Phi^{j} \frac{d x^{i}}{d x^{j}}}\right)\right] \Delta^{i} \Delta^{i}$

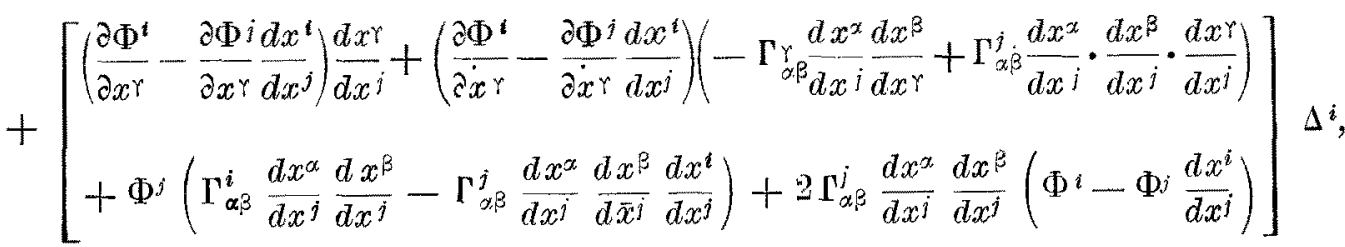

where $i$ and $j$ with $i \neq j$, are fixed, and $l \neq i, j$, varies over the remaining inlegers $1,2, \ldots, n$.

This proposition is derived from equations (7.1), (9.3), and (9.4).

By comparing (7.10) and (9.5), it is seen that every system $\Gamma$ of $\infty^{2 n-1}$ curvature trajectories $C$, is of the type $(G)$. However, not every system of the type $(G)$, is a system $\Gamma$ of $\infty^{2 n-1}$ curvature trajectories $O$.

It is remarked that not every complete physical system $S_{k}$ where $k \neq-1$, is a system $\Gamma$ of $\infty^{2 n-1}$ curvature trajecories $C$. For example, KasNer 
proved that in a Euclidean plane, a complete dynamical system $S_{0}$ is a system $\Gamma$ of $\infty^{3}$ curvature trajectories $C$ if and only if the corresponding positional field of force $\Phi$, is either central or parallel [10].

In a given positional field of force $\Phi$, a velocity family $F$ of $\infty^{2 n-2}$ veloeity curves $C$, is defined in the following manner. [11]. For such a curve $C$, it is understood that a particle of unit mass is constrained to move along it. A curve $C$ is a member of the velocity family $F$ if and only if a particle of unit mass, starting from any lineal element $E$ of $C$ with unit speed, describes a dynamical trajectory which initially osculates the curve $C$.

THeorem 9.2. - A velocity family $F$ of $\infty^{2 n-2}$ velocity curves $C$ is given by the intrinsic equations

$$
r N=1, \quad x^{i}=\Phi^{i}-T_{1} \frac{d x^{i}}{d s},
$$

where $i=1,2, \ldots, n$. The system of $(n-1)$ explicit ordinary differential equations, each of the second order, of a velocity family $F$ of $\infty^{2 n-2}$ velocity curves $C$, is

$$
\Delta^{i}=\left(\Phi^{i}-\Phi^{j} \frac{d x^{i}}{d x^{j}}\right)\left(g_{\alpha \beta} \frac{d x^{\alpha}}{d x^{j}} \frac{d x^{\beta}}{d x^{j}}\right)
$$

where $j$ is fixed, and $i \neq j$, varies over the remaining integers $1,2, \ldots, n$.

This result follows from equations (6.2), and (7.3).

A line of force $C$ is a velocity curve $C$ of a velocity family $F$ of $\infty^{2 n-2}$ velocity carves $C$, if and only if $C$ is a geodesic of the Riemannian space $\nabla_{n}$.

The center $\bar{x}$ of circular curvature at a given point $x$ of a curve $C$, is given by the set of equations

$$
\overline{x^{i}}=x^{i}+r^{2} x^{i}
$$

for $i=1,2, \ldots, n$. If there is a constrained motion along this curve $C$ for a given positional field of force $\Phi$, then

$$
\Phi_{i}\left(x^{i}-x^{i}\right)=r N
$$

Theorem 9.3. - Property A. A family $F$ of $\infty^{2 n-2}$ curves $C$ in a Riemannian space $V_{n}$, is a velocity family $F$ of $\infty^{2 n-2}$ velocity curves $C$ of a positional field of force $\Phi$, if and only if at each point $x$ of any curve $C$ of the family $F$, the force vector $\Phi$ is tangent to the osculating geodesic surface of $C$, and the center $\bar{x}$ of circular curvature of $C$, satisfies the condition

$$
\Phi_{i}\left(x^{i}-x^{i}\right)=1 \text {. }
$$


For, the first part of Property $A$, is equivalent to the set of $n$ conditions

$$
\Phi^{i}=T_{\mathbf{1}} \frac{d x^{i}}{d s}+r N x^{i}
$$

for $i=1,2, \ldots, n$. By (9.9), and the second part (9.10) of Property $A$, it is seen that $r N=1$. Substituting $r N=1$ into (9.11), it follows by (9.6), that Property $A$ characterizes a velocity family $F$ of $\infty^{2 n-2}$ velocity curves $C$ of a positional field of force $\Phi$.

The condition (9.10) states that if the tangent flat space $R_{n}$ of $n$ dimensions, of the Riemannian space $V_{n}$. is constructed at a point $x$ through which pass $\infty^{n-1}$ velocity curves $C$ of the velocity family $F$, then the corresponding centers $\bar{x}$ of circular curvature, describe a flat space $R_{n-1}$ of $(n-1)$ dimensions in $R_{n}$, which does not pass through the given point $x$.

Theorem 9.4. - A complete velocity system $S_{\infty}$ of $\infty^{2 n-1}$ curves $C$ of a positional field of force $\Phi$ in a Riemannian space $V_{n}$, is composed of the $\infty^{2 n-1}$ curvature trajectories $C$ of the velocity family $F$ of $\infty^{2 n-2}$ velocity curves $C$ of the same positional field of force $\Phi$.

This is a consequence of equations (6.2) and (9.6).

10. Conformal maps of Riemannian spaces. Consider two Riemannian spaces $V_{n}$ and $\bar{V}_{n}$ whose metrics are given by the two definite quadratic differential forms $d s^{2}=g_{i j} d x^{i} d x^{j}$, and $\overline{d s}^{2}=\bar{g}_{i j} d x^{i} d x^{i}$, where each one of the functions $g_{i j}, \bar{g}_{i j}$, is at least of class three in an $n$ dimensional region of points $x$. Two points of $V_{n}$ and $\bar{\nabla}_{n}$, are said to correspond if and only if they are represented by the same curvilinear coordinates $x=\left(x^{1}, x^{2}, \ldots, x^{n}\right)$, of the given region of points $x$. This establishes a point to point transformation $T$ between $\nabla_{n}$ and $\bar{V}_{n}$. Such a representation of $\bar{V}_{n}$ and $\nabla_{n}$, is called a cartogram $T$.

Let $\mu$ denote some positive scalar point function at least class three in the given region of point $x$. The cartogram $T$ is said to be conformal and the two Riemannian spaces $V_{n}$ and $\bar{V}_{n}$ are said to be conformally equivalent, if and only if $d s^{-}=\mu d s$, for some such positive scalar point function $\mu$. Then $\bar{g}_{i j}=\mu^{2} g_{i j}$, and $\bar{g}^{i j}=\frac{1}{\mu^{2}} g^{i j}$. In this case, $\bar{V}_{n}$ is called a conformal image of $V_{n}$.

If $\bar{V}_{n}$ is a conformal image of $V$, then $d \bar{s}=\mu d s$, where $\mu>0$, is a positive scalar point function. If $\mu>0$, is a constant, then $\bar{V}_{n}$ is said to be a homothetic image of $V_{n}$, and the cartogram is called a homothetic transformation $T$. In particular, if $\mu=1$, then $\bar{V}_{n}$, is called an isometric image of $V_{n}$, or $\bar{V}_{n}$ is said to be applicabile to $V_{n}$, and the cartogram is termed an isometric correspondence $T$. 
It is easy to see that a cartogram $T$ between two Riemannian spaces $V_{n}$ and $\bar{V}_{n}$, is conformal if and only if $T$ preserves the magnitude of the angle between any two curves $C_{1}$ and $C_{2}$ which pass through an arbitrary point $x$.

Consider a conformal cartogram $T$ between two Riemannian spaces $V_{n}$ and $\bar{V}_{n}$ so that $\bar{V}_{n}$ is a conformal image of $V_{n}$. Then $\overline{d s}=\mu d s$ where $\mu>0$, a positive scalar point function. Clearly

$$
\bar{g}_{i j}=\mu^{2} g_{i j}, \quad \bar{g}^{i j}=\frac{1}{\mu^{2}} g^{i j} .
$$

In this conformal cartogram $T$, the Christoffel symbols of the first kind, are related as follows

$$
\bar{\Gamma}_{i j ; k}=\mu^{2} \Gamma_{i j ; k}+\mu\left(g_{i k} \frac{\partial \mu}{\partial x^{j}}+g_{j k} \frac{\partial \mu}{\partial x^{i}}-g_{i j} \frac{\partial \mu}{\partial x^{k}}\right)
$$

and the Christoffel symbols of the second kind, correspond by the equations

$$
\bar{\Gamma}_{j k}^{i}=\frac{g^{i l}}{\mu^{2}} \bar{\Gamma}_{j k ; l}=\Gamma_{j k}^{i}+\frac{1}{\mu}\left(\delta_{i}^{i} \frac{\partial \mu}{\partial x^{k}}+\delta_{k}^{i} \frac{\partial \mu}{\partial x^{j}}-g_{j k} g^{i l} \frac{\partial \mu}{\partial x^{i}}\right) .
$$

Under this conformal cartogram $T$, the absolute total derivatives with with respect to are length of a contravariant vector $\lambda^{i}$, correspond as follows

$$
\frac{\bar{D} \lambda^{i}}{d s}=\frac{1}{\mu} \frac{D \lambda^{i}}{d s}+\frac{1}{\mu^{2}} \frac{\partial \mu}{\partial x^{k}} \lambda^{i} \frac{d x^{k}}{d s}+\frac{1}{\mu^{2}} \frac{\partial \mu}{\partial x^{j}} \lambda j \frac{d x^{i}}{d s}-\frac{1}{\mu^{2}} \frac{\partial \mu}{\partial x^{l}} g_{j k} g^{i l} \lambda^{j} \frac{d x^{h}}{d s}
$$

Theorem 10.1 - Let a Riemannian space $V_{n}$ be mapped into a Riemannian $\bar{V}_{,}$by a conformal transformation $T$. Under $T$, a curve $C$ in $V_{n}$, is converted into a curve $\bar{C}$ in $\bar{V}_{n}$. The vector geodesic curvatures $x$ and $\bar{\kappa}$ at corresponding points of $C$ and $C$, are related by the expressions

$$
\overline{x^{i}}=\frac{1}{\mu^{2}} x^{i}+\frac{1}{\mu^{3}} \frac{d \mu}{d s} \frac{d x^{i}}{d s}-\frac{1}{\mu^{3}} g^{i l} \frac{\partial \mu}{\partial x^{l}},
$$

where $i=1,2, \ldots, n$.

This result is found by substituting $\lambda^{i}=\frac{d x^{i}}{d s}=\frac{1}{\mu} \frac{d x^{i}}{d s}$, into (10.4). 
11. Natural families. The $\infty^{2 n-2}$ extremals $C$ in a Riemannian space $V_{n}$, of the variation problem

$$
\int_{1}^{2} \mu d s=\operatorname{minimum},
$$

are said to form a natural family $F$ in $V_{n}$. This is equivalent to saying that a natural family $F$ of $\infty^{2 n-2}$ curves $C$ in a Riemannian space $V_{n}$, is a conformal image in $V_{n}$, of the family $\bar{F}$ of $\infty^{2 n-2}$ geodesies $\bar{C}$ of a Riemannian space $\bar{V}_{n}$, which is a conformal representation of $V_{n}$. Some. times, the $\infty^{2 n-2}$ curves $C$ of a natural family $F$ in a Riemannian space $V_{n}$, are called conform-geodesics [12].

THEOREM 11.1. The intrinsic differential equations a natural family $F$ of $\infty^{2 n-2}$ curves $C$ in Riemannian space $V_{n}$, are

$$
x^{i}=\frac{1}{\mu} g^{i l} \frac{\partial \mu}{\partial x^{i}}-\frac{1}{\mu} \frac{d \mu}{d s} \frac{d x^{i}}{d s},
$$

where $i=1,2, \ldots, n$.

For, upon setting $\overline{x^{i}}=0$, in equations (10.5), the conditions (11.2), are found.

From the above proposition, it follows that a natural family $F$ is composed of the $\infty^{2 n-2}$ geodesics in the Riemannian space $V_{n}$, if and only if $\mu>0$, is a constant. Therefore, a conformal map $T$ of a Riemannian space $V_{n}$ onto a Riemannian space $\bar{V}_{n}$, converts the family $F$ of $\infty^{2 n-2}$ geodesics $C$ in $V_{n}$, into the family $\bar{F}$ of $\infty^{3 n-2}$ geodesies $\bar{C}$ in $\bar{V}_{n}$, if and only if $T$ is a homothetic transformation.

THeorem 11.2. A family $F$ of $\infty^{2 n-2}$ curves $C$ in a Riemannian space $V_{n}$, is a natural family $F$ for which the associated scalar point function $\mu>0$, is not a constant, if and only if it is a velocity family $F$ of $\infty^{2 n-2}$ velocity curves $C$ of a conservative field of force $\Phi=-\frac{\partial V}{\partial x}$, for which $V$ is $a$ potential function. In this case, the relations between the scalar point function $\mu>0$, and the potential function $V$, are

$$
V=\log \frac{1}{\mu}, \quad \mu=e^{-V}
$$

For, by comparing (9.6) and (11.2), it follows that a natural family $F$ of $\infty^{2 n-2}$ curves $C$ in $V_{n}$, for which the associated scalar point function $\mu>0$, is not a constant, is a velocity family $F$ of $\infty^{2 n-2}$ velocity curves 
$C$ of a conservative field of force $\Phi=-\frac{\partial V}{\partial x}$, for which a potential function $V$, is $V=\log 1 / \mu$.

Conversely, a velocity family $F$ of $\infty^{2 n-2}$ velocity curves $C$ of a conservative field of force $\Phi=-\frac{\partial V}{\partial x}$, with potential function, $V$, is a natural family $F$. The associated scalar point function is $\mu=e^{-V}$. Clearly this is positive, and is not constant.

It is clear that.if a positional field of force $\Phi$ in a Riemannian space $V_{n}$, is not conservative, then the velocity family $F$ of $\infty^{2 n-2}$ velocity curves $C$, is not natural family $F$.

Theorem 11.3. Under a conformal map $T$ of a Riemannian space $V_{n}$ onto a Riemannian space $\bar{V}_{n}$, every velocity family $F$ of $\infty^{2 n-2}$ velocity curves $C$ in $V_{n}$, is converted into a velocity family $\bar{F}$ of $\infty^{2 n-2}$ velocity curves $\bar{C}$ in $\bar{V}_{n}$. The relations between the corresponding fields of force $\Phi$ and $\bar{\Phi}$, are

$$
\bar{\Phi}^{i}=\frac{1}{\mu^{2}} \Phi^{i}-\frac{1}{\mu^{3}} g^{i l} \frac{\partial \mu}{\partial x^{l}} ; \quad \bar{\Phi}_{i}=\Phi_{i}-\frac{1}{\mu} \frac{\partial \mu}{\partial x^{i}} ;
$$

for $i=1,2, \ldots, n$.

For, in $\bar{V}_{n}$, a velocity family $\bar{F}$ of $\infty^{2 n-2}$ vetocity curves $\bar{C}$, is given by the conditions

$$
\overline{\chi^{i}}=\bar{\Phi}^{i}-\left(\bar{g}_{\alpha \beta} \bar{\Phi}^{\alpha} \frac{d x^{\beta}}{d \bar{s}}\right) \frac{d x^{i}}{\overline{d s}}
$$

Substituting (10.1) and (10.5) into these equations, it is found that this family $\bar{F}$ in $\bar{V}_{n}$, corresponds by the conformal map $T$, to the velocity family $F$ of $\infty^{2 n-2}$ velocity curves $C$ in $V_{n}$, which is given by the equations

$$
x^{i}=\left(\mu^{2} \bar{\Phi}^{i}+\frac{1}{\mu} g^{i l} \frac{\partial \mu}{\partial x^{i}}\right)-\left(\mu^{2} g_{\alpha \beta} \bar{\Phi}^{\alpha} \frac{d x^{\beta}}{d s}+\frac{1}{\mu} \frac{d \mu}{d s}\right) \frac{d x^{i}}{d s}
$$

From these equations, the relations (11.4) are deduced.

By the last three propositions, it is an immediate consequence that under a conformal map $T$ of a Riemannian space $V_{n}$, onto a Riemannian space $\bar{V}_{n}$, every natural family $F$ of $\infty^{2 n-2}$ curves $C$ in $V_{n}$, is converted into a natural $\bar{F}$ of $\infty^{3 n-2}$ curves $\bar{C}$ in $\bar{V}_{,}$.

In the above paragraph, let $F$ be represented as a natural velocity family $F$ of $\infty^{2 n-2}$ velocity curves $C$ of a conservative field of force $\Phi=-\frac{\partial V}{\partial x}$, with a potential function $V$, in the Riemannian space $V_{n}$, 
and let $\bar{F}$ be depicted as a natural velocity family $\bar{F}$ of $\infty^{2 n-z}$ velocity curves $\bar{C}$ of a conservative field of force $\bar{\Phi}=-\frac{\partial \bar{V}}{\partial x}$, with a potential function $\vec{V}$, in the Riemannian space $\bar{V}_{n}$. Then under the conformal map $T$ of the Riemannian space $V_{n}$ onto the Riemannian space $\bar{V}_{n}$, the two potencial functions $V$ and $\bar{V}$, are related by the single condition

$$
\bar{\gamma}=V+\log \mu
$$

except for an additive constant $c$ of integration.

12. Conservative physical systems $S_{k}$ where $k \neq-1, \infty$ [13]. A physical system $S_{k}$ where $k \neq-1, \infty$, of a conservative field of force $\Phi=-\frac{\partial V}{\partial x}$, with potential function $V$, is termed a conservative physical system $S_{k}$ where $k \neq-1, \infty$, of $\sim^{2 n-1}$ trajectories $C$, in a Riemannian space $V_{n}$.

Theorem 12.1 - If $L=T-(1+k) V=\frac{m}{2} g_{\alpha \beta} \dot{x}^{\alpha} x^{\beta}-(1+k) V$, the Lagrangian differential equations of a conservative physical system $S_{k}$ where $k \neq-1, \infty$, of $\infty^{2 n-1}$ trajectories $C$, in Riemannian space $V_{n}$, are.

$$
\frac{d}{d t}\left(\frac{\partial L}{\partial \dot{x}^{i}}\right)-\frac{\partial L}{\partial x^{i}}-\frac{k \frac{d V}{d t}}{2[L+(1+k) V]} \frac{\partial L}{\partial x^{i}}=0,
$$

where $i=1,2, \ldots, n$.

For, substitute $\Phi_{i}=-\frac{\partial V}{\partial x^{i}}$, into equations (5.5). By use of the relation $T=L+(1+k) V$, the equations (5.5) become the Lagrangian differential equations (12.1) of a conservative physical system $S_{k}$ where $k \neq-1, \infty$.

For $k=0, L$ becomes the Lagrangian function $L=T-V$. Then the equations (12.1) become the Lagrangian equations (12.1) of a conservative dynamical system $S_{0}$ of $\infty^{2 n-1}$ dynamical trajectories $O$.

Theorem $12.2-A$ conservative physical system $S_{k}$ where $k \neq-1, \infty$, of $\infty^{2 n-1}$ curves $O$ in a Riemannian space $V_{n}$, is given by the $n$ ordinary second order differential equations

$$
x^{i}=-\frac{(1+k)}{2(E-V)}\left(g^{i l} \frac{\partial V}{\partial x^{i}}-\frac{d V}{d s} \frac{d x^{i}}{d s}\right)
$$


for $i=1,2, \ldots, n$, where the total energy $E$, is a constant of integration.

For the equations (5.2) become the equations (12.2) upon substituting $\Phi_{i}=-\frac{\partial V}{\partial x^{i}}$, and $m v^{2}=2(E-V)=(1+k) r N$.

THEOREM 12.3. - Corresponding to the value of the total energy constant $E$, a conservative physical system $S_{k}$ where $k \neq-1, \infty$, can be separated into $\infty^{\prime}$ natural families $S_{k}(E)$ each one of which is composed of $\infty^{2 n-2}$ trajectories C. Any such natural faniy $S_{k}(E)$ consists of the $\sim^{2 n-2}$ extremals $C$ of the variation problem

$$
\int_{1}^{2} v^{1+k} d s=\text { minimum }
$$

For, by (12.2), the associated positive scalar function $\mu>0$, of the natural family $S_{k}(E)$, obeys the conditions

$$
\frac{1}{\mu} \frac{\partial \mu}{\partial x^{i}}=-\frac{(1+k)}{2(E-V)} \frac{\partial V}{\partial x^{i}}
$$

for $i=i, 2, \ldots, n$. Exept for a multiplicative constant $c>0$, of integration, it is seen that $\mu=\left[\frac{2}{m}(E-V)\right]^{\frac{(1+k)}{2}}=v^{1+k}$.

For $k=0$, the integral (12.3), is the action integral of HaMuLToN. Thus the dynamical family $S_{0}(E)$ of $\infty^{2 n-2}$ actual dynamical trajectories $C$, is composed of the $\infty^{2 n-2}$ extremals $C$ of the variation problem

$$
\int_{1}^{2} v d s=\text { minimum. }
$$

For $k=1$, the catenary family $S_{1}(E)$ of $\infty^{2 n-2}$ virtual catenaries $C$, consists of the $\infty^{2 n-2}$ extremals $C$ of the variation problem

$$
\int_{1}^{2} v^{2} d s=\text { minimum }
$$

Finally for $k=-2$, the brachistochrone family $S_{-2}(E)$ of $\infty^{2 n-2}$ actual brachistochrones $C$, is the set of $\infty^{2 n-2}$ extremals $C$ of the variation problem

$$
T=\operatorname{Time}=\int_{1}^{2} d t=\int_{1}^{2} v^{-1} d s=\text { minimum. }
$$


By (12.4), any natural family $F$ of $\infty^{2 n-2}$ curves $C$ for which the associated positive scalar point function $\mu>0$, is not a constant, can be depicted as a physical family $S_{k}(E)$ of $\infty^{2 n-2}$ trajectories $C$, where $k \neq-1, \infty$. Any associated potential function $V$ is of the form

$$
V=E-a \mu^{2 /(1+k)}
$$

where $a>0$, is an arbitrary constant.

THEOREM 12.4. - Under a conformal map $T$ of a Riemannian space $V_{n}$ onto a Riemannian space $\bar{V}_{n}$, any physical family $S_{k}(E)$ where $k \neq-1, \infty$, of $\infty^{2 n-2}$ trajectories $C$ in $V_{n}$, is converted into a physical family $\overline{S_{k}}(\bar{E})$ of $\infty^{2 n-2}$ trajectories $\bar{C}$ in $\overline{V_{n}}$, with the same value of $k \neq-1, \infty$. The relation between the two potential functions $V$ and $\bar{V}$ of the two corresponding conser. vative fields of force $\Phi=-\frac{\partial V}{\partial x}$, and $\bar{\Phi}=-\frac{\partial \bar{V}}{\partial x}$, is

$$
\bar{V}=a \mu-\frac{2}{(1+k)}(V-E)+\bar{E}
$$

where $a>0$, is an arbitrary positive consant.

For, let the conformal map $T$ of the Riemannian space $V_{n}$ onto the Riemannian space $\bar{V}_{n}$ be represented by $d \bar{s}=\mu d s$. The physical family $S_{k}(E)$ where $k \neq-1, \infty$, can be visualized as the natural family $F$ of $\infty^{2 n-2}$ extremals $C$ of the variation problem: $\int_{1}^{2} v d s=$ minimum. Similarly, the physical family $\bar{S}_{k}(\bar{E})$ where $k \neq-1, \infty$, can be depicted as the natural family $\bar{F}$ of $\infty^{2 n-2}$ extremal $\bar{C}$ of the variation problem: $\int_{1}^{2} \bar{v} d \bar{s}=$ minimum. Under the conformal map $T$, it follows that $\bar{\nu}=\nu / \mu$. Therefore, by means of the relation (12.8), it is found that

$$
\bar{V}=\bar{E}-a \mu^{-\frac{2}{(1+k)} y} \frac{2}{1+k}=a \mu^{-\frac{2}{1+k}}(V-E)+\bar{E}
$$

where $a>0$, is an arbitrary positive constant.

THEOREM 12.5. - A conformal transformation $T$ between two Riemannian space $V_{n}$ and $\bar{V}_{n}$, is a homothetic correspondence $T$, if and only if T converts every conservative complete physical system $S_{k}$ with $k \neq-1, \infty$, of $\infty^{2 n-1}$ trajectories $C$ in $V_{n}$, into a conservative complete physical system $S_{k}$ with the same $k \neq-1, \infty$, of $\infty^{2 n-1}$ trajectories $\bar{C}$ in $\bar{V}_{n}$. When the conformal transforma. tion $T$ is not homothetic, that is, when the associated positive scalar point 
function $\mu>0$, is not a constant, then under $T$, there is one and only one conservative complete physical system $S_{k}$ with $k \neq-1, \infty$, of $\infty^{2 n-1}$ trajecto. ries $C$ in $V_{n}$, which is carried into a conservative complete physical system $\bar{S}_{k}$ with the same $k \neq-1, \infty$, of $\infty^{2 n-1}$ trajectories $\bar{C}$ in $\bar{V}_{n}$. For this latter case, the associated potential functions $V$ and $\bar{V}$, are

$$
V=-\frac{\bar{E}}{a} \mu^{\frac{2}{1+k}}, \quad \bar{V}=-a E \mu^{-\frac{2}{1+k}}
$$

where $a \neq 0$, is an arbitrary constant, and $E \neq 0, \vec{E} \neq 0$, are the two corresponding constant total energies.

For, by (12.9), it is seen that a conformal map $T$ carries every conservative complete physical system $S_{k}$ with $k \neq-1, \infty$, of $\infty^{2 n-1}$ trajectories $C$ in $V_{n}$, into a conservative complete physical system $\bar{S}_{k}$ with the same $k \neq-1, \infty$, of $\infty^{2 n-1}$ trajectories $\bar{C}$ in $\overline{V_{n}}$, if and only if $\mu>0$, is a con. stant. This means that $T$ is a homothetic correspondence.

Consider the case where the associated positive scalar point function $\mu>0$, of the conformal transformation $T$, is not a constant. That is, $T$ is not a homothetic map. For this case, the two conservative complete physical system $S_{k}$ with $k \neq-1, \infty$, and $\bar{S}_{k}$ with the same $k \neq-1, \infty$, are given by the two conservative fields of force $\Phi=-\frac{\partial W}{\partial x}$, and $\bar{\Phi}=-\frac{\partial \bar{W}}{\partial x}$, for which the corresponding potential functions $W$ and $\bar{W}$, are

$$
W=-\frac{a}{\bar{E}} V, \quad \bar{W}=-\frac{1}{a \bar{E}} \bar{V}
$$

where $a \neq 0$, and $E \neq 0, \bar{E} \neq 0$, are the total energies, that appear in (12.9). From (12.9), these two potential functions $W$ and $W$ are related by the condition

$$
\bar{W}-\mu-\frac{2}{1+k}=\frac{\bar{E}}{a E} \mu-\frac{2}{1+k}\left(W-\mu \frac{2}{1+k}\right) .
$$

Since $\frac{\bar{E}}{a E}$, is an arbitrary constant and since $\mu^{-\frac{2}{1+k}}>0$, is not a constant, it follows that

$$
W=\mu^{2 /(1+k)}, \quad \bar{W}=\mu^{-2 /(1+k)} .
$$

Substituting (12.14) into (12.12), the equations (12.11) are found.

This completes the proof of Theorem 12.5. 
13. Conservative velocity systems $S_{\infty}$. A velocity system $S_{\infty}$ of a conservative field of force $\Phi=-\frac{\partial V}{\partial x}$, with potential function $V$, is said to be a conservative velocity system $S_{\infty}$ of $\infty^{2 n-1}$ trajectories $C$, in a Riemannian space $V_{n}$.

Theorem 13.1 . The Lagrangian equations of a conservative velocity system $S_{\infty}$ of $\infty^{2 n-1}$ trajectories $C$, in a Riemannian space $V_{n}$, are

$$
m v_{0}^{2}\left[\frac{d}{d t}\left(\frac{\partial T}{\partial \dot{x}^{i}}\right)-\frac{\partial T}{\partial x^{i}}\right]+2 T \frac{\partial V}{\partial x^{i}}+\frac{\bar{d} t}{2 T}\left(m v_{0}^{2}-2 T\right) \frac{\partial T}{\partial \dot{x}^{i}}=0
$$

for $i=1,2, \ldots, n$.

This follows by substituting $r N=m v_{0}^{2}$, and $\Phi_{i}=-\frac{\partial V}{\partial x^{i}}$, into equations (6.5).

THEOREM 13.2 - A conservative velocity system $S_{\infty}$ of $\infty^{2 n-1}$ velocity curves $C$ in a Riemannian space $V_{n}$, is given by the $n$ ordinary second order differential equations

$$
x^{i}=-\frac{1}{m v_{0}^{2}}\left(g^{i l} \cdot \frac{\partial V}{\partial x^{2}}-\frac{d V}{d s} \frac{d x^{i}}{d s}\right)
$$

for $i=1,2, \ldots, n$. where $m v_{0}^{2}>0$, is a constant of integration.

This is obtained from equations (6.6) upon substituting $r N=m v_{0}^{2}$, and $\Phi_{i}=-\frac{\partial V}{\partial x^{i}}$.

THEOREM 13.3 - Corresponding to the value of the constant speed $v_{0}>0$, a conservative velocity system $S_{\infty}$, can be segregated into $\infty^{1}$ natural families $S_{\infty}\left(v_{0}\right)$, each one of which is composed of $\infty^{2 n-2}$ velocity curves $C$. Any such natural family $S_{\infty}\left(v_{0}\right)$ consist of the $\infty^{2 n-2}$ extremals $C$ of the variation problem

$$
\int_{i}^{2} e^{\frac{v^{2}}{2 v_{0^{2}}^{2}}} d s=\text { minimum. }
$$

For, upon comparing (11.2) and (13.2), it is found that $\mu=e-V / m v^{2}$, except for a multiplicative constant $c \neq 0$, of integration. Since $V=E-\frac{m}{2} v^{2}$, it is seen that the $\mu$ can take the form $\mu=e^{v^{2} / 2 v_{0}{ }^{2}}$. 
Evidently the variation problem (13.3), is equivalent to the variation problem

$$
\int_{1}^{2} e^{-\frac{V}{m v_{0}^{2}}} d s=\text { minimum. }
$$

THEOREM 13.4 - Under a conformal map $T$ of a Riemannian space $V_{n}$ onto a Riemannian space $\bar{V}_{n}$, any velocity family $S_{\infty}\left(v_{0}\right)$ of $\infty^{2 n-2}$ velocity curves $C$ in $V_{n}$, is converted into a velocity family $\bar{S}_{\infty}\left(\bar{v}_{0}\right)$ of $\infty^{2 n-2}$ velocity curves $\bar{C}$ in $\bar{V}_{n}$. The relation between the two potential functions $V$ and $\bar{V}$ of the two corresponding conservative fields of force $\Phi=-\frac{\partial V}{\partial x}$, and $\bar{\Phi}=-\frac{\partial \bar{V}}{\partial x}$, is

$$
\frac{\bar{V}}{\overline{\bar{m} \bar{v}_{0}^{2}}}=\frac{V}{m v_{0}^{2}}+\log \mu
$$

except for an additive constant $c$.

For, let the conformal map $T$ between the two Riemannian spaces $V_{n}$ and $\bar{V}_{n}$, be represented by $\overline{d s}=\mu d s$. In $\bar{V}_{n}$, the velocity family $\bar{S}_{\infty}\left(\bar{v}_{0}\right)$ is composed of the $\infty^{2 n-2}$ extremals $\bar{C}$ of the variation problem

$$
\int_{1}^{2} e^{-\frac{\bar{V}}{\bar{m} \bar{v}_{0}^{2}}} d \bar{s}=\int_{1}^{2} e^{-\frac{\bar{V}}{\bar{m} \bar{v}_{0}^{2}}} \mu d s=\text { minimum. }
$$

This can be visualized as a variation problem in $V_{n}$, for which the extremals are the $\infty^{2 n-2}$ velocity curves $C$ of the velocity family $S_{\infty}\left(v_{0}\right)$, provided that

$$
e^{-\frac{\bar{V}}{\bar{m} \bar{v}_{0}^{2}} \mu}=e^{-\frac{V}{m v_{0}^{2}}}
$$

except for an arbitrary multiplicative constant $e^{-c}>0$. Solving this equation for $\bar{V} / \vec{m} \bar{v}_{0}^{2}$, the condition (13.5), is found.

Theorem 13.5 - A conformal transformation $T$ between two Riemannian spaces $V_{n}$ and $\bar{V}_{n}$, is a homothetic map $T$, if and only if $T$ carries every conservative complete velocity system $S_{\infty}$ of $\infty^{2 n-1}$ velocily curves $C$ in $V_{n}$, into a conservative complete velocity system $\bar{S}_{\infty}$ of $\infty^{2 n-1}$ velocity curves $\bar{C}$ in $\bar{V}_{n}$. When the conformal transformation $T$ is not homothetic, that is, when the associated positive scalar point function $\mu>0$, is not a constant, then under $T$, there is one and only one conservative complete velocity 
system $S_{\infty}$ of $\infty^{2 n-1}$ velocity curves $C$ in $V_{n}$, which is carried into a conservative complete velocity system $\bar{S}_{\infty}$ of $\infty^{2 n-1}$ velocity curves $\bar{C}$ in $\bar{V}_{n}$. In this case, the associated potential functions $V$ and $\bar{\nabla}$, are

$$
V=a \log \mu, \quad \bar{V}=b \log \mu,
$$

where $a \neq 0, b \neq 0$, are two arbitrary constants for which

$$
\frac{b}{\bar{m} \bar{v}_{0}^{2}}=\frac{a}{m v_{0}^{2}}+1
$$

This is proved by means of equation (13.5) of Theorem 13.4.

14. The infinitesimal contact transformation $T$ of a Riemannian space $V_{n}$. Let the $p_{i}$ denote the covariant form of the unit tangent of any curve $C$ in a Riemannian space $V_{n}$. Then

$$
\frac{d x^{i}}{d s}=g^{i j} p_{j} ; \quad p_{i}=g_{i j} \frac{d x^{j}}{d s}
$$

By equations (2.2), (2.3), and (14.1), the covariant form $x_{i}$ of the vector curvature $x$ of a curve $C$ in a Riemannian space $V_{n}$, is

$$
x=x_{i}=g_{i j} \frac{d^{2} x^{j}}{d s^{2}}+g_{i j} \Gamma_{\alpha \beta}^{j} \frac{d x^{\alpha}}{d s} \frac{d x^{\beta}}{d s}=\frac{d p_{i}}{d s}-\frac{1}{2} \frac{\partial g_{\alpha \beta}}{\partial x^{i}} \frac{d x^{\alpha}}{d s} \frac{d x^{\beta}}{d s}
$$

Therefore

$$
x=x_{i}=\frac{d p_{i}}{d s}-\frac{1}{2} \frac{\partial g_{\alpha \beta}}{\partial x^{i}} g^{\times i} g^{\beta k} p_{j} p_{k}
$$

$$
=\frac{d p_{i}}{d s}+\frac{1}{2} g_{\alpha \beta} g^{\alpha j} \frac{\partial g^{\beta k}}{\partial x^{i}} p_{j} p_{k} \equiv \frac{d p_{i}}{d s}+\frac{1}{2} \frac{\partial g^{j k}}{\partial x^{i}} p_{j} p_{k}
$$

THEOREM 14.1 - The characteristic function $\mathrm{I}=\mathrm{T}(\boldsymbol{x} ; p)$, of a Riemannian space $V_{n}$, is

$$
\Gamma=\Gamma(x ; p)=\left(g^{\times \beta} p_{\alpha} p_{\beta}\right)^{1 / 2}
$$

This characteristic function $\Gamma$ is linear homogeneous in the $p_{i}$, and has the value unity along any curve $C$ in $V_{n}$. In terms of this characteristic function $\Gamma=\Gamma(x ; p)$, the contravariant form $\frac{d x^{i}}{d s}$ of the unit tangent vector 
$\tau_{1}=\frac{d x}{d s}$, and the covariant form $x_{i}$ of the vector geodesic curvature $x$ of a curve $C$, are

$$
\frac{d x^{i}}{d s}=\frac{\partial \Gamma}{\partial p_{i}}=g^{i j} p_{j}, \quad x_{i}=\frac{d p_{i}}{d s}+\frac{\partial \Gamma}{\partial x^{i}}=\frac{d p_{i}}{d s}+\frac{1}{2} \frac{\partial g^{j k}}{\partial x^{i}} p_{j} p_{k}
$$

For, it is seen from (14.4), that $\Gamma=\Gamma(x ; p)$, is linear homogeneous in the $p_{i}$. Also $\Gamma=\Gamma(x ; p)$, is the magnitude of the unit tangent vector $\tau_{1}=\frac{d x}{d s}$, of the curve $C$, and hence has the value unity along C. Along such a curve $C$, it is evident that

$$
\begin{gathered}
\frac{d x^{i}}{d s}=g^{i j} p_{j}=\frac{1}{\Gamma} \frac{\partial \Gamma}{\partial p_{i}}=\frac{\partial \Gamma}{\partial p_{i}}, \\
x_{i}=\frac{d p_{i}}{d s}+\frac{1}{2} \frac{\partial g^{j k}}{\partial x^{i}} \cdot p_{j} p_{k}=\frac{d p_{i}}{d s}+\frac{1}{\Gamma} \frac{\partial \Gamma}{\partial x^{i}}=\frac{d p_{i}}{d s}+\frac{\partial \Gamma}{\partial x^{i}} .
\end{gathered}
$$

The infinitesimal contact transformation $T$ of the Riemannian space $V_{n}$, is

$$
\delta x^{i}=\frac{\partial \Gamma}{\partial p_{i}} \delta p=g^{i j} p_{j} \delta s, \quad \delta p_{i}=-\frac{\partial \Gamma}{\partial x^{i}} \delta s=-\frac{1}{2} \frac{\partial g^{j k}}{\partial x^{i}} p_{j} p_{k} \delta s
$$

This is given the Lie symbol $U F$ or the Porsson parenthesis $(\Gamma, F)$, by means of the expression

$$
\begin{aligned}
& U F=(\Gamma, F)=\frac{\partial \Gamma}{\partial p^{i}} \frac{\partial F}{\partial x^{i}}-\frac{\partial \Gamma}{\partial x^{i}} \frac{\partial F}{\partial p_{i}} \\
& =\left(g^{i j} p_{j}\right) \frac{\partial F}{\partial x^{j}}-\frac{1}{2}\left(\frac{\partial g^{j k}}{\partial x^{i}} p_{j} p_{k}\right) \frac{\partial F}{\partial p_{i}} .
\end{aligned}
$$

The infinitesimal contact transformation $T$ or the symbol $U F$, generates the one-parameter dilatation group $\Delta$, of the Riemannian space $V_{n}$. The path curves $C$ of this one-parameter dilatation group $\Delta$, obey the differential equations

$$
\frac{d x^{i}}{d s}=\frac{\partial \Gamma}{\partial p_{i}}=g^{i j} p_{j}, \frac{d p_{i}}{d s}=-\frac{\partial \Gamma}{\partial x^{i}}=-\frac{1}{2} \frac{\partial g^{j k}}{\partial x^{i}} p_{j} p_{k}
$$

Clearly, the path curves $C$ form the family $F$ of $\infty^{2 n-2}$ geodesic $C$ of the Riemannian space $V_{n}$. 
A spatial element $E$ in the Riemannian space $V_{n}$, is composed of a point $x$ and a spatial direction of $(n-1)$ dimensions passing through this point $x$. A spatial direction of $(n-1)$ dimensions at a point $x$, can be defined by a non-zero covariant vector $p=p_{i}$, which is orthogonal to it. Therefore a spatial element $E$ can be defined by a set of coordinates $(x ; p)$, where at least one $p_{i}$, is not zero. If two sets of coordinates $(x ; p)$ and $(x ; \bar{p})$, define the same spatial element $E$, then $\bar{x}=x$, and $\bar{p}=\rho p$, where $\rho \neq 0$, is an arbitrary factor of proportionality.

Any dilatation $D_{k}$ of the one-parameter dilatation group $\Delta$ of the Rie. mannian space $\nabla_{n}$, converts every spatial element $E$ into a spatial element $\bar{E}$ such that the points $x$ and $\bar{x}$ of $E$ and $\bar{E}$, are on the same geodesic $C$, the spatial directions of $E$ and $\bar{E}$ are orthogonal to this geodesic $C$ at the points $x$ and $\bar{x}$, respectively, and the geodesic distance along this geodesic $C$ between the two points $x$ and $\bar{x}$ of $E$ and $\bar{E}$, is the constant $k \geqq 0$.

A family of $\infty^{\prime}$ surfaces, $\nabla(x)=$ constant, each of $(n-1)$ dimensions, is called a parallel family in the Riemannian space $\nabla_{n}$, if and only if this family is orthogonal to a set of $\infty^{n-1}$ geodesics $C$ in $V_{n}$. Such a family is a parallel family if and only if it is left invariant by the one-parameter dilatation group $\Delta$ of the Riemannian space $\nabla_{n}$.

A family of $\infty^{1}$ surfaces, $V(x)=$ constant, each of $(n-1)$ dimensions, is a parallel family in the Riemannian space $V_{n}$, if and only if the function $V(x)$ can be chosen so that it obeys the first order Hamilton-Jacobi parial differential equation

$$
g^{i j} \frac{\partial V}{\partial x^{i}} \frac{\partial V}{\partial x^{j}}=1
$$

for this Riemannian space $V_{n}$.

15. The infinitesimal contact transformation $T$ of a natural family $F$ in a Riemannian space $\nabla_{n}$. Let a Riemannian space $\bar{V}_{n}$, be a conformal representation of the Riemannian space $V_{n}$. Then $d \bar{s}=\mu d s$, where the associated positive scalar point function is $\mu>0$. Consequently, $\bar{g}_{i j}=\mu^{2} g_{j}$ and $\bar{g}^{i j}=\frac{1}{\mu^{2}} g^{i j}$.

For a curve $\bar{C}$ in $\bar{V}_{n}$, the covariant form $\bar{p}_{i}$ of the unit tangent vector $\bar{\mu}_{1}$, is given by the relations

$$
\overrightarrow{p_{i}}=\overline{g^{i j}} \frac{d x_{j}}{\overrightarrow{d s}}=\mu g_{i j} \frac{d x^{j}}{d s}=\mu p_{i}
$$

$$
\frac{d x^{i}}{d s}=g^{i j} p_{j}=\frac{1}{\mu} g^{i j} \bar{p}_{j} .
$$


The characteristic function $\bar{\Gamma}=\bar{\Gamma}(x ; \bar{p})$, of the Riemannian space $\bar{\nabla}_{n}$, is

$$
\bar{\Gamma}=\bar{\Gamma}(x ; \bar{p})=\frac{1}{\mu}\left(g^{\alpha \beta} \bar{p}_{\alpha} \bar{p}_{\beta}\right)^{1 / 2}=\frac{1}{\mu} \Gamma(x ; \bar{p}) .
$$

It is evident that $\bar{\Gamma}=\bar{\Gamma}(x ; \bar{p})=\frac{1}{\mu} \Gamma(x ; \bar{p})=\Gamma(x ; p)$, since $\bar{p}_{i}=\mu p_{i}$.

Since $\Gamma(x ; p)=1$, along a curve $C$ of $V_{n}$, then $\Gamma(x ; \bar{p})=\mu$, along the eurve $\bar{C}$ of $\bar{V}_{n}$, which is the conformal image of the curve $C$ of $\nabla_{n}$.

The covariant form $\bar{x}_{i}$ of the vector geodesic curvature $\bar{x}$ of a curve $\bar{C}$ in $\bar{\nabla}_{n}$, is

$$
\begin{gathered}
\overline{x_{i}}=\frac{d \overline{p_{i}}}{d \bar{s}}+\frac{\partial \bar{\Gamma}}{\partial x^{i}}=\frac{1}{\mu}\left(\frac{d \bar{p}_{i}}{d s}-\frac{1}{\mu} \Gamma \frac{\partial \mu}{\partial x^{i}}+\frac{\partial \Gamma}{\partial x^{i}}\right) \\
=\frac{1}{\mu}\left(\frac{d \overline{p_{i}}}{d s}-\frac{\partial \mu}{d x^{i}}+\frac{\partial \Gamma}{\partial x^{i}}\right) \\
=\frac{1}{\mu}\left(\frac{d \bar{p}_{i}}{d s}-\frac{\partial \mu}{\partial x^{i}}+\frac{1}{2 \mu} \frac{\partial g^{j k}}{\partial x^{i}} \bar{p}_{j} \bar{p}_{k}\right) .
\end{gathered}
$$

The family $F$ of $\infty^{2 n-2}$ geodesics $\bar{C}$ in the Riemannian space $\vec{V}_{n}$, is depicted as the natural family $F$ of $\infty^{2 n-2}$ curves $C$ in the Riemannian space $\nabla_{n}$. The infinitesimal contact transformation $T$ of this family $F$ of $\infty^{2 n-2}$ curves $C$ in the Riemannian space $V_{n}$, is that of the Riemannian space $\bar{V}_{n}$. [14].

THeOREM 15.1. - The characteristic function of the infinitesimal contact transformation $T$ of a natural family $F$ of $\infty^{2 n-2}$ curves $C$ of a Riemannian space $V_{n}$, is $\bar{\Gamma}=\bar{\Gamma}(x ; \bar{p})=\frac{1}{\mu} \Gamma(x ; \bar{p})$, where $\Gamma(x ; \bar{p})=\left(g^{\alpha \beta} \bar{p}_{\alpha} \bar{p}_{\beta}\right)^{1 / 2}$, is the characteristic function of the original Riemannian space $\nabla_{n}$. This infinitesimal contact transformajion $T$, is given by the equations

$$
\delta x^{i}=\frac{\partial \Gamma}{\partial \bar{p}_{i}} \delta s=\frac{1}{\mu}\left(g^{i j} \bar{p}_{j}\right) \delta s,
$$

$$
\delta \bar{p}_{i}=\left(\frac{\partial \mu}{\partial x^{i}}-\frac{\partial \Gamma}{\partial x^{i}}\right) \delta s=\left(\frac{\partial \mu}{\partial x^{i}}-\frac{1}{2 \mu} \frac{\partial g^{j k}}{\partial x^{i}} \bar{p}_{j} \bar{p}_{k}\right) \delta s .
$$


Also this is given by the symbol $U F$, namely

$$
\begin{aligned}
U F & =(\Gamma, F)+\frac{\partial \mu}{\partial x^{i}} \frac{\partial F}{\partial \bar{p}_{i}}=\frac{\partial \Gamma}{\partial \bar{p}_{i}} \frac{\partial F}{\partial x^{i}}+\left(\frac{\partial \mu}{\partial x^{i}}-\frac{\partial \Gamma}{\partial x^{i}}\right) \frac{\partial F}{\partial \bar{p}_{i}} \\
& =\frac{1}{\mu}\left(g^{i j} \bar{p}_{j}\right) \frac{\partial F}{\partial x^{i}}+\left(\frac{\partial \mu}{\partial x^{i}}-\frac{1}{2 \mu} \frac{\partial g^{j k}}{\partial x^{i}} \bar{p}_{j} \bar{p}_{k}\right) \frac{\partial F}{\partial \bar{p}_{i}} .
\end{aligned}
$$

This follows from the previous discussion.

Theorex 15.2. - The differential equations of a natural family $F$ of $\infty^{2 n-2}$ curves $C$ in a Riemannian space $V_{n}$, may be written in the form

$$
\begin{aligned}
& \frac{d x^{i}}{d s}=\frac{\partial \Gamma}{\partial \bar{p}_{i}}=\frac{1}{\mu} g^{i j} \bar{p}_{j}, \\
& \frac{d \bar{p}_{i}}{d s}=\frac{\partial \mu}{\partial x^{i}}-\frac{\partial \Gamma}{\partial x^{i}}=\frac{\partial \mu}{\partial x^{i}}-\frac{1}{2 \mu} \frac{\partial g^{j k}}{\partial x^{i}} \bar{p}_{j} \bar{p}_{k},
\end{aligned}
$$

for $i=1,2, \ldots, n$.

This a consequence of the definition of the infinitesimal contact transformation $T$ of a natural family $F$ of $\infty^{2 n-2}$ curves $C$ in the Riemannian space $V_{n}$.

The preceding infinitesimal contact transformation $T$, or its corresponding symbol $U F$, generates a one-parameter group $\bar{\Delta}$ of contact transformations $\bar{D}_{k}$ in the Riemannian space $\nabla_{n}$. This one-parameter group $\bar{\Delta}$ is the one-parameter dilatation group $\bar{\Delta}$ of the Riemannian space $\bar{\nabla}_{n}$, which is the conformal representation of the given Riemannian space $V_{n}$.

Any contact transformation $\bar{D}_{k}$ of the preceding one-parameter group $\bar{\Delta}$ in the Riemannian space $\nabla_{n}$, sends every spatial element $E$ into a spatial element $\bar{E}$ such that the points $x$ and $\bar{x}$ of $E$ and $\bar{E}$, are on the same curve $C$ of the natural family $F$, the spatial directions of $E$ and $\bar{E}$ are orthogonal to this carve $C$ at the points $x$ and $\bar{x}$ respectively, and the value of the integral: $\int_{x}^{\bar{x}} \mu d s$, evaluated along this curve $C$ between the two points $x$ and $\bar{x}$ of $E$ and $\bar{E}$, is the constant $k \geqq 0$.

Theorem 15.3. A family of $\infty^{1}$ surfaces, $V(x)=$ constant, each of $(n-1)$ dimensions, is orthogonal to a set of $\infty^{n-1}$ curves $C$ of a natural family $F$ of the Riemannian space $V_{n}$ if and only if the function $V(x)$ can be so chosen 
that it obeys the first order Hamilton-Jacobi partial differential equation

$$
g^{i j} \frac{\partial V}{\partial x^{i}} \frac{\partial V}{\partial x^{j}}=\mu^{2}
$$

For, each transformation $\bar{D}_{k}$ of the one-parameter group $\bar{\Delta}$, discussed above, is a contact transformation $\vec{D}_{k}$.

The Riemannian space $V_{n}$ may be visualized as an optical medium for which the index of refraction is the positive scalar point function $\mu>0$. The set of $\infty^{2 n-2}$ light rays $C$ of this optical medium is composed of the $\infty^{2 n-2}$ extremals $C$ of the variation problem

$$
T=\operatorname{Time}=\int_{1}^{2} \mu d s=\text { minimum }
$$

Therefore, this set of $\infty^{2 n-2}$ light rays $C$, is a natural family $F$ of $\infty^{2 n-2}$ curves $C$.

In this optical medium, a set of $\infty^{x}$ wave fronts is given by the equation $\nabla(x)=$ constant, where the function $V(x)$ is so chosen that it obeys the first order HaMiLToN-JACOBI partial differential equation (15.7). Each surface of this set of $\infty^{1}$ wave fronts, given by the equation $\nabla(x)=$ constant, is of dimension $(n-1)$.

16. The Lagrangian and Hamitonian equations of a natural family $F$ of $\infty^{2 n-2}$ curves $C$. It is supposed that a particle of mass $m>0$, is constrained to move alone a curve $C$ of a natural family $F$ of $\infty^{2 n-2}$ curves $C$, such that it is influenced by a conservative field of force $\Phi=-\frac{\partial V}{\partial x}$, for which a potential function is $\nabla=V(x)$.

For such a particle of mass $m>0$, the vector $q$ is given in the contra. variant form $q^{i}$ and in the covariant form $q_{i}$ by the formulas

$$
q^{i}=m \frac{\mu}{v} \frac{d x^{i}}{d t} ; \quad q_{i}=m \frac{\mu}{v} g_{i,} \frac{d x^{j}}{d t}=m \bar{p}_{i}
$$

Evidently, $\Gamma(x ; \bar{p})=\frac{1}{m} \Gamma(x ; q)$. It is clear that $\Gamma(x ; q)=m \mu$, along such a curve $C$.

Under these conventions, the differential equations (15.6) of the natural 
family $F$ of $\infty^{2 n-2}$ curves $C$, can be written in the form

$$
\begin{aligned}
& \frac{d x^{i}}{d t}=v \frac{\partial \Gamma(x ; q)}{\partial q_{i}}=\frac{v}{m \mu} g^{i j} q_{j}=\frac{v}{m \mu} q^{i}, \\
& \frac{d q_{i}}{d t}=-v \frac{\partial \Gamma(x ; q)}{\partial x^{i}}+m v \frac{\partial \mu}{\partial x^{i}}=-\frac{v}{2 m \mu} \frac{\partial g^{j k}}{\partial x^{i}} q_{j} q_{k}+m v \frac{\partial \mu}{\partial x^{i}} .
\end{aligned}
$$

THEOREM 16.1 - The absolute total derivative $\frac{D q_{i}}{d t}$ of the covariant vector $q_{i}$ with respect to the time $t$, along any one of the $\infty^{2 n-2}$ curves $C$ of this natural family, $F$, is

$$
\frac{D q_{i}}{d t}=\frac{d q_{i}}{d t}-\Gamma_{\beta i}^{\Upsilon} q_{\Upsilon} \frac{d x^{\beta}}{d t}=m v \frac{\partial \mu}{\partial x^{i}},
$$

for $i=1,2, \ldots, n$.

This is proved by using equations (16.2). It is seen that

$$
\begin{aligned}
& \frac{D q_{i}}{d t}=\frac{d q_{i}}{d t}-\Gamma_{\beta i}^{\gamma} q_{\gamma} \frac{d x^{\beta}}{d t}=\frac{d q_{i}}{d t}-\frac{m \mu}{v} g_{\alpha \gamma} \Gamma_{\beta i}^{\gamma} \frac{d x^{\alpha}}{d t} \frac{d x^{\beta}}{d t} \\
& =\frac{d q_{i}}{d t}-\frac{m \mu}{v} \Gamma_{\beta i ; \alpha} \frac{d x^{\alpha}}{d t} \frac{d x^{\beta}}{d t}=\frac{d q_{i}}{d t}-\frac{m \mu}{2 v} \frac{\partial g_{\alpha \beta}}{\partial x^{i}} \frac{d x^{\alpha}}{d t} \frac{d x^{\beta}}{d t}
\end{aligned}
$$

\section{Consequently}

$$
\begin{aligned}
& \frac{D q_{i}}{d t}=\frac{d q_{i}}{d t}-\frac{v}{2 m \mu} g^{\alpha j} g^{\beta k} \frac{\partial g_{\alpha \beta}}{\partial x^{i}} q \cdot q_{k}=\frac{d q_{i}}{d t}+\frac{v}{2 m \mu} g^{\alpha j} g_{\alpha \beta} \frac{\partial g^{\beta k}}{\partial x^{i}} q_{j} q_{k} \\
& =\frac{d q_{i}}{d t}+\frac{v}{2 m \mu} \frac{\partial g^{j k}}{\partial x_{i}} q_{j} q_{k}=m v \frac{\partial \mu}{\partial x^{i}} .
\end{aligned}
$$

This completes the derivation of the equations (16.3).

Theorem 16.2 - If the Lagrangian function $L$ is defined as

$$
L=\frac{\mu}{v}(T-\nabla+E)-E
$$


then the set of $\infty^{2 n-2}$ extremals $C$ of the variation problem

$$
\int_{1}^{2} L d t=\int_{1}^{2}\left[\frac{\mu}{v}(T-V+E)-E\right] d t=\text { minimum }
$$

is the natural family $F$ of $\infty^{2 n-2}$ curves $C$. Therefore, the system of Lagrangian equations for this natural family $F$ of $\infty^{2 n-2}$ curves $C$, is

$$
\frac{d}{d t}\left(\frac{\partial L}{\partial \dot{x}^{i}}\right)-\frac{\partial L}{\partial x^{i}}=\frac{\mu}{v}\left[\frac{d}{d t}\left(\frac{\partial T}{\partial \dot{x}^{i}}\right)-\frac{\partial T}{\partial x^{i}}\right]+\left[\frac{d}{d t}\left(\frac{\mu}{v}\right)\right] \frac{\partial T}{\partial \dot{x}^{i}}-m v \frac{\partial \mu}{\partial x^{i}}=0,
$$

for $i=1,2, \ldots, n$.

For, if $L$ denotes the Lagrangian function (16.6), then

$$
\begin{aligned}
\frac{d}{d t}\left(\frac{\partial L}{\partial \dot{x}^{i}}\right)- & \frac{\partial L}{\partial x^{i}}=\frac{\mu}{v} \frac{d}{d t}\left(\frac{d T}{\partial \dot{x}^{i}}\right)+\left[\frac{d}{d t}\left(\frac{\mu}{v}\right)\right] \frac{\partial T}{\partial \dot{x}^{i}}-\frac{\mu}{v} \frac{\partial T}{\partial x^{i}} \\
& +\frac{\mu}{v} \frac{\partial V}{\partial x^{i}}-\frac{2 T}{v^{2}}\left(v \frac{\partial \mu}{\partial x^{i}}-\mu \frac{\partial v}{\partial x^{i}}\right) \\
& =\frac{\mu}{v}\left[\frac{d}{d t}\left(\frac{\partial T}{\partial \dot{x}^{i}}\right)-\frac{\partial T}{\partial x^{i}}\right]+\left[\frac{d}{d t}\left(\frac{\mu}{v}\right)\right] \frac{\partial T}{\partial \dot{x}^{i}}-m v \frac{\partial \mu}{\partial x^{i}}
\end{aligned}
$$

Since the EuLER-Lagrange differential equations for the set of $\infty^{2 n-2}$ extremals $C$ of the variation problem (16.7), are $\frac{d}{d t}\left(\frac{\partial L}{\partial \dot{x}^{i}}\right)-\frac{\partial L}{\partial \dot{x}^{i}}=0$, for $i=1,2, \ldots, n$, it follows that the set of $\infty^{2 n-2}$ extremals $C$ of the variation problem (16.7), is given by the system of Lagrangian equations (16.8)

By substituting (16.1) into (16.3), the system of Lagrangian equations (16.8). is obtained. Consequently, the set of $\infty^{2 n-2}$ extremals $C$ of the variation problem (16.7), is our given natural family $F$ of $\infty^{2 n-2}$ curves $C$.

The proof of Theorem 16.2 , is complete.

From equations (16.1) and (16.2), the kinetic energy $T$ of a particle of mass $m>0$, traversing such a curve, $C$, is

$$
T=\frac{m}{2} g_{i j} \frac{d x^{i}}{d t} \frac{d x^{j}}{d t}=\frac{v^{2}}{2 m \mu^{2}} g^{i j} q_{i} q_{j}=\frac{v^{2}}{2 m \mu^{2}}[\Gamma(x ; q)]^{2}
$$

THEOREM 16.3 - If the Hamltonian function $H$ is taken as

$$
H=H(x ; q)={ }_{v}^{\mu}(T+V-E)+E,
$$


then the natural family $F$ of ${ }^{2 n-2}$ curves $C$, is composed of the set of $\infty^{2 n-2}$ integral solutions $C$ of the system of Hamiltonian equations

$$
\frac{d x^{i}}{d t}=\frac{\partial H}{\partial q_{i}}, \frac{d q_{i}}{d t}=-\frac{\partial H}{\partial x^{i}}
$$

for $i=1,2, \ldots, n$. This Hamiltonian function $H$ is the constant total energy $E$, along any curve $C$ of the $\infty^{2 n-2}$ curves $C$ of the natural family $F$.

For, when the Hamiltonia function $H$ is given by (16.11), then subject to the condition that $T+V=E$, the expanded forms of the Hamiltonian equations (16.12), are

$$
\begin{gathered}
\frac{d x^{i}}{d t}=\frac{\partial H}{\partial q_{i}}=\frac{\mu}{v} \frac{\partial T}{\partial q_{i}}=\frac{v}{m \mu} g^{i j} q_{j}=\frac{v}{m \mu} q^{i}, \\
\frac{d q_{i}}{d t}=-\frac{\partial H}{\partial x^{i}}=-\frac{\mu}{v} \frac{\partial T}{\partial x^{i}}-\frac{\mu}{v} \frac{\partial V}{\partial x^{i}}-(T+V-E) \frac{\partial}{\partial x^{i}}\left(\frac{\mu}{v}\right) \\
=-\frac{\mu}{v} \frac{\partial T}{\partial x^{i}}+m \mu \frac{\partial v}{\partial x^{i}} \\
=-\frac{v}{2 m \mu} \frac{\partial g^{j k}}{\partial x^{i}} q_{j} q_{k}+\frac{1}{m \mu^{2}}\left(v \frac{\partial \mu}{\partial x^{i}}-\mu \frac{\partial v}{\partial x^{i}}\right)[\Gamma(x ; q)]^{2}+m \mu \frac{\partial v}{\partial x^{i}} \\
=-\frac{v}{2 m \mu} \frac{\partial g^{j k}}{\partial x^{i}} q_{j} q_{k}+m v \frac{\partial \mu}{\partial x^{i}},
\end{gathered}
$$

By equations (16.2), it follows that (16.12) are the Hamiltoniann equations of the natural family $F$ of $\infty^{2 n-2}$ eurves $C$ for which the Hamiltonian function $H$ is given by (16.11). Thus the proposition is proved.

It is remarked that the Lagrangian function $L$ and the Hamiltonian function $H$ are related by the equations

$$
H+L=\frac{2 \mu}{v} T, \quad H-L=-m v \mu+2 E
$$

17. The infinitesimal contact transformation $T$ associated with a conservative physical family $S_{k}(E)$ where $k \neq-1, \infty$, of $\infty^{2 n-2}$ trajectories $C$. By means of Theorem 12.3 and Theorem 15.1, the following proposition may be established.

THeonem 17.1 - The conservative physical family $S_{k}(E)$ where $k \neq-1$, $\infty$, is composed of the $\infty^{2 n-2}$ trajectories $C$ of the one-parameter group $\bar{\Delta}$ 
of contact transformations $\bar{D}_{k}$, generated by the infinitesimal contact transformation $T$ whose characteristic function $\bar{\Gamma}=\bar{\Gamma}(x ; \bar{p})$, is

$$
\bar{\Gamma}=\bar{\Gamma}(x ; \bar{p})=\frac{1}{v^{1+k}} \Gamma\left(x ; \overline{p)}=\left[\left.\frac{g_{\alpha \beta} \bar{p}_{\alpha} \bar{p}_{\beta}}{\left\{\frac{2}{m}(E-V)\right\}^{1+k}}\right|^{1 / 2}\right.\right.
$$

This is the infinitesimal contact transformation $T$ associated with the conservative physical family $S_{k}\left(E_{)}\right)$where $k \neq-1, \infty$, of $\infty^{2 n-2}$ trajectories $C$.

This infinitesimal contact transformation $T$, is given by the equations

$$
\begin{aligned}
\delta x^{i} & =v^{-1-k}\left(g^{i j} \bar{p}_{j}\right) \delta s \\
\delta \bar{p}_{i} & =\left[(1+k) v^{k} \frac{\partial v}{\partial x^{i}}-\frac{1}{2} v^{-1-k} \frac{\partial g^{j k}}{\partial x^{i}} \bar{p}_{j} \bar{p}_{k}\right] \delta s .
\end{aligned}
$$

Also it given by the symbol $U F$, namely

$$
\begin{aligned}
& U F=v^{-1-k}\left(g^{i j} \bar{p}_{j}\right) \frac{\partial F}{\partial x^{i}} \\
& +\left[(1+k) v^{k} \frac{\partial v}{\partial x^{i}}-\frac{1}{2} v^{-1-k} \frac{\partial g^{j k}}{\partial x^{i}} \bar{p}_{j} \bar{p}_{k}\right] \frac{\partial F}{\partial \bar{p}_{i}}
\end{aligned}
$$

Whe $k=0$, this reduces to the infinitesimal contact transformation $T$ of mechanies studied by Lie and Vessiot.

THeorem 17.2 - If the Lagrangian function $L$ is defined as

$$
L=v^{k}(T-V+E)-E
$$

then the set of $\infty^{2 n-2}$ extremals $C$ of the variation problem

$$
\int_{1}^{2} L d t=\int_{1}^{2}\left[v^{k}(T-V+E)-E\right] d t=\operatorname{minimum}
$$

is the conservative physical family $S_{k}(E)$ where $k \neq-1, \infty$, of $\infty^{2 n-2}$ trajectories $C$.

This is a consequence of Theorem 12.3 and Theorem 16.2. 
When $k=0$, the above proposition reduces to the well-known result concerning a conservative dynamical family $S_{0}(E)$ of $\infty^{2 n-2}$ trajectories $C$.

TheOREM 17.3 - The Hamiltonian function $H$ of a conservative physical family $S_{k}(E)$ where $k \neq-1, \infty$, of $\infty^{2 n-2}$ trajectories $C$, is

$$
H=H(x ; q)=v^{h}(T+V-E)+E .
$$

This follows from Theorem 12.3 and Theorem 16.3.

When $k=0$, this results becomes the well-known one concerning a conservative dynamical family $S_{0}(E)$ of $\infty^{2 n-2}$ trajectories $C$.

18. The infinitesimal contact transformation $T$ associated with a conservative velocity family $S_{\infty}\left(v_{0}\right)$ of $\infty^{2 n-2}$ velocity carves $C$. By Theorem 13.3 and Theorem 15.1, the following result can be established.

TheоRem 18.1 - The conservative velocity family $S_{\infty}\left(v_{0}\right)$ of $\infty^{2 n-2}$ velocity curves $C$, is composed of the $\infty^{2 n-2}$ trajectories $C$ of the one parameter group $\bar{\Delta}$ of contact transformations $\bar{D}_{k}$, generated by the infinitesimal contact transformation $T$ whose characteristic function $\bar{\Gamma}=\bar{\Gamma}(x ; \bar{p})$, is

$$
\bar{\Gamma}=\bar{\Gamma}(x ; \bar{p})=e^{-\frac{v^{2}}{2 v_{0}^{2}} \Gamma(x ; \bar{p})=\left[e^{-\frac{v^{2}}{v_{0}^{2}}} g^{\alpha \beta} \bar{p}_{\alpha} \bar{p}_{\beta}\right] .^{\frac{1}{2}}}
$$

This is the infinitesimal contact transformation $T$ associated with a conservative velocity family $S_{\infty}\left(v_{0}\right)$ of $\infty^{2 n-2}$ velocity curves $C$.

This infinitesimal contact transformation $T$ is given by the equations

$$
\begin{aligned}
& \delta x^{i}=e^{-\frac{v^{2}}{2 v_{0}^{2}}}\left(g^{i j} \bar{p}_{j}\right) \delta s, \\
& \delta p_{i}^{-}=\left(\frac{v}{v_{0}^{2}} e^{\frac{v^{2}}{2 v_{0}^{2}} \frac{\partial v}{\partial x^{i}}}-\frac{1}{2} e^{\left.-\frac{v^{2}}{2 v_{0}^{2}} \frac{\partial g^{j k}}{\partial x^{i}} \bar{p}_{j} \bar{p}_{k}\right) \delta s .}\right.
\end{aligned}
$$

Also it is given by the symbol $U F$, namely

$$
\begin{aligned}
& U F=e^{-\frac{v^{2}}{2 v_{0}^{2}}}\left(g^{i j} \bar{p}_{j}\right) \frac{\partial F}{\partial x^{i}} \\
& +\left(\frac{v}{v_{0}^{2}} e^{\frac{v^{2}}{2 v_{0}^{2}}} \frac{\partial v}{\partial x^{i}}-\frac{1}{2} e^{\left.-\frac{v^{2}}{2 v_{0}^{2}} \frac{\partial g^{j k}}{\partial x^{i}} \bar{p}_{j} \bar{p}_{k}\right) \frac{\partial F}{\partial \bar{p}_{i}}}\right.
\end{aligned}
$$


Theorem 18.2. - If the Lagrangian function $L$ is taken to be the expression

$$
L=\frac{1}{v} e^{\frac{v^{2}}{2 v_{0}^{2}}(T-V+E)-E,}
$$

then the set of $\infty^{2 n-2}$ extremals $C$ of the variation problem

$$
\int_{1}^{2} L d t=\int_{1}^{2}\left[\frac{1}{v} e^{\frac{v^{2}}{2 v_{3}^{3}}}(T-V+E)-E\right] d t=\text { minimum }
$$

is the conservative velocity family $S_{\infty}\left(v_{0}\right)$ of $\infty^{2 n-2}$ velocity curves $C$.

This follows from Theorem 13.3 and Theorem 16.2.

THeorem 18.3. - The Hamiltonian function $H$ of a conservative velocity family $S_{\infty}\left(v_{0}\right)$ of $\infty^{2 n-2}$ velocity corves $C$, is

$$
H=H(x ; q)=\frac{1}{v} e^{\frac{v^{2}}{2 v_{0}^{2}}(T+V-E)+E .}
$$

This is a consequence of Theorem 13.3 and Theorem 16.3.

19. Inflnitesimal contact transformations $T$ [15]. Consider a spatial element transformation $T$ in a space of dimension $n$, given by a system of $2 n$ equatons

$$
X^{i}=X^{i}(x ; p) ; \quad P_{i}=P_{i}(x ; p) ;
$$

for $i=1,2, \ldots, n$, such that each one of the $2 n$ functions $X^{i}(x ; p) ; P_{i}(x ; p)$; is of class $m \geqq 3$, and the Jacobian matrix is of rank $2 n$, in a certain $2 n$ dimensional region of values $(x ; p)$.

A transformation $T$ of this kind, is said to be a homogeneous contact transformation $T$, or more simply, a contact transformation $T$, if and only the differential expression $p_{i} d x^{i}$, is invariant under $T$.

A spatial element correspondence $T$ is a contact transformation $T$ if and only if the following set of $2 n$ conditions is valid, namely

$$
P_{\alpha} \frac{\partial X^{\alpha}}{\partial x^{i}}=p_{i} ; \quad P_{\alpha} \frac{\partial X^{\alpha}}{\partial p_{i}}=0
$$

for $i=1,2, \ldots, n$.

An infinitesimal spatial element transformation $T$, is defined by a set of $2 n$ conditions of the form

$$
\delta x^{i}=X^{i}-x^{i}=\xi^{i}(x ; p) \delta s ; \delta p_{i}=P_{i}-p_{i}=\eta_{i}(x ; p) \delta s
$$


for $i=1,2, \ldots, n$, where $\delta s$, is an infinitesimal, and each one of the $2 n$ functions $\xi^{i}(x ; p) ; \eta_{i}(x ; p)$; is of the class $m \geqq 2$, with at least one being not identically zero, in a certain $2 n$ dimensional region of values $(x ; p)$.

A given infinitesimal spatial element transformation $T$, generate a oneparameter group $G_{1}$ of spatial element correspondences $T_{k}$, and conversely.

The preceding one-parameter group $G_{1}$ of spatial element correspondences $T_{k}$, may be represented uniquely not only by its infinitesimal spatial element transformation $T$ as given by the equations (19.3), but also by its Lie symbol $U F$. This symbol $U F$ is given by the expression

$$
U F=\xi^{i} \frac{\partial F}{\partial x^{i}}+\eta_{i} \frac{\partial F}{\partial p_{i}}
$$

For this one-parameter group $G_{1}$, there is a set of $\infty^{2 n-2}$ path spatial element series $\Sigma$, which is the collection of the $\infty^{2 n-2}$ integral solutions $\Sigma$ of the system of $2 n$ first order ordinary differential equations

$$
\frac{d x^{i}}{d s}=\xi^{i}(x ; p) ; \quad \frac{d p_{i}}{d s}=\eta_{i}(x ; p)
$$

for $i=1,2, \ldots, n$. This set is said to be the fundamental system of ordinary differential equations of the given one-parameter group $G_{1}$.

If every $\xi^{i}$ is independent of all of the $p^{\prime} s$, and if at lcast one $\xi^{i}$ is not identically zero, then the path curves $C$ of the path spatial element series $\Sigma$ of the one-parameter group $G_{1}$, form a set of $\infty^{n-1}$ path curves $C$.

Suppose that at least one $\xi^{i}$ is not independent of all of the $p^{\prime} s$. The direction determined by the vector $\delta x$ with the contravariant components $\delta x^{i}$ such that this vector $\delta x$ has its initial point $x$ at that of the spatial element $(x ; p)$ and its terminal point $X$ at that of the spatial element $(X ; P)$ where these are related by the equations (19.3) of the infinitesimal spatial element transformation $T$, is said to be transversal to the direction of the covariant vector $p_{i}$.

Under these assumption, the transversality law defined by the infinitesimal spatial element transformation $T$, is given by the set of $n$ equations

$$
\frac{d x_{i}}{d s}=\xi^{i}(x ; p)
$$

for $i=1,2, \ldots, n$.

The infinitesimal spatial element transformation $T$ given by equations (19.3), is an infinitesimal contact transformation $T$ if and only if the following 
set of $2 n$ conditions are satisfied, namely

$$
p_{\alpha} \frac{\partial \xi^{x}}{\partial x^{i}}+\eta_{i}=0 ; \quad p_{\alpha} \frac{\partial \xi^{\alpha}}{\partial p_{i}}=0
$$

for $i=1,2, \ldots, n$.

Under these conditions, set $p_{x} \xi^{x}=\Gamma(x ; p)$. It is found that this characteristic function $\Gamma=\Gamma(x ; p)$, of the infinitesimal contact transformation $T$, is a non-constant function of class $m \geqq 2$. in the given $2 n$ dimensional region of values $(x ; p)$, and is homogeneous of degree one in the $p^{\prime} s$. Moreover

$$
\xi^{i}=\frac{\partial \Gamma}{\partial p_{i}} ; \quad \eta_{i}=-\frac{\partial \Gamma}{\partial x^{i}} ;
$$

for $i=1,2, \ldots, n$.

Therefore, an infinitesimal contact transformation $T$ is defined by a system of $2 n$ equations

$$
\delta x^{i}=X^{i}-x^{i}=\frac{\partial \Gamma}{\partial p_{i}} \delta s ; \delta p_{i}=P_{i}-p_{i}=-\frac{\partial \Gamma}{\partial x^{i}} \delta s
$$

for $i=1,2, \ldots, n$, where the characteristic function $\Gamma=\Gamma(x ; p)$, is not constant, and is homogeneous of degree one in the $p^{\prime} s$.

This infinitesimal contact transformation $T$ generates a one-parameter group $\Delta$ of contact transformations $D_{k}$.

It is seen that a one-parameter group $\Delta$ of contact transformations $D_{k}$, is represented uniquely, not only by its infinitesimal contact transformation $T$, but also by its LIE symbol $U F$. This symbol $U F$ is given by the PoIsson parenthesis

$$
U F=(\Gamma, F)=\frac{\partial \Gamma}{\partial p_{\alpha}} \frac{\partial F}{\partial x^{\alpha}}-\frac{\partial \Gamma}{\partial x^{x}} \frac{\partial F}{\partial p_{\alpha}} .
$$

The infinitesimal contact transformation $T$ is a point transformation $T$ if and only if its characteristic functions $\Gamma=\Gamma(x ; p)$, is linear integral in the $p^{\prime} s$. This infinitesimal point transformation $T$, generates a one-parameter group $\Delta$ of point correspondences $D_{k}$. For this one-parameter point group $\Delta$ there is a set of $\infty^{n-1}$ trajectories $C$.

Henceforth, let the infinitesimal contact transformation $T$ be not a point transformation $T$. This is equivalent to saying that the characteristic function $\Gamma=\mathrm{I}(x ; p)$, is not linear integral in the $p^{\prime} s$. The corresponding one-parameter group $\Delta$, is composed of contact transformations $D_{k}$, which are not point correspondences $D_{k}$. 
For, the preceding one-parameter group $\Delta$, there is a set of $\infty^{2 n-2}$ trajectories $C$. This is the collection of $\infty^{2 n-2}$ integral solutions $C$ of the fundamental system of $2 n$ first order ordinary differential equations

$$
\frac{d x^{i}}{d s}=\frac{\partial \Gamma}{\partial p_{i}} ; \quad \frac{d p_{i}}{d s}=-\frac{\partial \Gamma}{\partial x^{i}} ;
$$

for $i=1,2, \ldots, n$. Along each one of the $\infty^{2 n-2}$ trajectories $C$, the charecteristic function $\Gamma=\Gamma(x ; p)$, is constant.

A non-singular trajectory $C$ is an integral solution $C$ of (19.11), along which the characteristic function $\Gamma=\Gamma(x ; p)$, is not zero. Thus the $p^{\prime} s$ may be so chosen that the characteristic function $\Gamma=\Gamma(x ; p)$, is unity along any non-singular trajectory $C$ of the set of $\infty^{2 n-2}$ trajectories $C$.

If the characteristic function $\Gamma=\Gamma(x ; p)$, is not linear integral in the $p^{\prime} s$, the transversality, law of the infinitesimal contact transformation $T$, is given by the set of $n$ equations

$$
\frac{d x^{i}}{d s}=\frac{\partial \Gamma}{\partial p_{i}}
$$

for $i=1,2, \ldots, n$.

20. The commutator $\left(U_{1}, U_{2}\right) F$ two infinitesimal contact transformations $U_{1} F$ and $U_{2} F$ [16]. Two distinct infinitesimal contact transformations $T_{1}$ and $T_{2}$, can be denoted by the two symbols $U_{1} F=\left(\Gamma_{1}, F\right)$ and $U_{2} F=\left(\Gamma_{2}, F\right)$, where the two corresponding characteristic functions $\Gamma_{1}=\Gamma_{1}(x ; p)$, and $\Gamma_{2}=$ $=\Gamma_{2}(x ; p)$, are not non-zero constant multiples of one another. Then the commutator or alternant $\left(U_{1}, U_{2}\right) F$, as given by the equation

$$
\left(U_{1}, U_{2}\right) F=\left(U_{1} U_{2}\right) F-\left(U_{2} U_{1}\right) F
$$

represents an infinitesimal contact transformation $T_{3}$.

The characteristic function $\Gamma_{3}=\Gamma_{3}(x ; p)$ of the infinitesimal contact transformation $\left(U_{1}, U_{2}\right) F$, is given by the PoIsson parenthesis

$$
\Gamma_{3}=\left(\Gamma_{1}, \Gamma_{2}\right)=\frac{\partial \Gamma_{1}}{\partial p_{\alpha}} \frac{\partial \Gamma_{2}}{\partial x^{\alpha}}-\frac{\partial \Gamma_{1}}{\partial x^{\alpha}} \frac{\partial \Gamma_{2}}{\partial p_{\alpha}}
$$

If $\Gamma_{1}=\Gamma_{1}(x ; p)$, and $\Gamma_{2}=\Gamma_{2}(x ; p)$, are each of class $m \geqq 3$, then $\Gamma_{3}=\Gamma_{3}(x ; p)$, is of class $m-1 \geq 2$, in the $2 n$ dimensional region of values $(x ; p)$. Of course, it is evident that $\Gamma_{3}=\Gamma_{3}(x ; p)$, is homogeneous of degree unity, in the $p^{\prime} s$. 
Let $U F$ and $\bar{U} F$ denote two distinct infinitesimal contact transformations $T$ and $\bar{T}$ such that neither one is a point correspondence. Then neither one of the two associated characteristic functions $\Gamma=\Gamma(x ; \bar{p})$, and $\bar{\Gamma}=\overline{\mathrm{T}}(x ; \bar{p})$, is linear integral in the $\bar{p}^{\prime} s$. Of course, neither characteristic function is a non-zero constant multiple of the other.

THEOREM 20.1. - The two distinct infinitesimal-contact transformations $U F$ and $\bar{U} F$, possess the same transversality law if and only if the two associated characteristic functions $\mathrm{\Gamma}=\mathrm{\Gamma}(x ; \bar{p})$, and $\bar{\Gamma}=\bar{\Gamma}(x ; \bar{p})$, are related as follows

$$
\bar{\Gamma}=\bar{\Gamma}(x ; \bar{p})=\frac{1}{\mu(x)} \Gamma(x ; \bar{p})=\frac{1}{\mu} \Gamma
$$

where $\mu(x)$ is at least of class two and never vanishes in a certain $n$ dimensional region of points $x$.

For, by (19.12), the two distinct infinitesimal contact transformations $U F$ and $\overline{U F}$, have the same transversality law if and only if

$$
\frac{\partial \bar{\Gamma}}{\partial \bar{p}_{i}} / \frac{\partial \Gamma}{\partial \bar{p}_{i}}=\frac{\partial \bar{\Gamma}}{\partial \bar{p}_{j}} / \frac{\partial \Gamma}{\partial \bar{p}_{j}} \quad \text {, for } i, j=1,2, \ldots, n
$$

Hence $\overline{\mathrm{N}}=F(x ; \mathbf{I})$ by the theory of Jacobians. Since $\mathrm{\Gamma}=\mathrm{T}(x ; \bar{p})$, and $\overline{\mathrm{T}}=$ $=\bar{\Gamma}(x ; p)$, are each homogeneous of degree one in the $\bar{p}$ s, it follows by EULER's theorem on homogeneus functions that

$$
\bar{p}_{i} \frac{\partial \bar{\Gamma}}{\partial \bar{p}_{i}}=\bar{p}_{i} \frac{\partial \Gamma}{\partial \bar{p}_{i}} \frac{\partial F}{\partial \Gamma}=\Gamma \frac{\partial F}{\partial \Gamma}=F
$$

Thus $F=\frac{1}{\mu(x)} \Gamma$, so that $\bar{\Gamma}=\bar{\Gamma}\left(x ; \bar{p}_{)}=\frac{1}{\mu(x)} \Gamma(x ; \bar{p})=\frac{1}{\mu} \Gamma\right.$. Because of the dif. ferentiability assumptions, it is seen that $\mu(x)$ is at least of class two and is never zero in an $n$ dimensional region of points $x$.

Let $U F, \bar{U}_{1} F, \bar{U}_{2} F$, denote three distinct infinitesimal contact transfor. mations $T, \overline{T_{1}}, T_{2}$, such that neither one is a point correspoodence. Then each one of the three associated characteristic funetions

$\Gamma=\Gamma(x ; \bar{p}), \quad \bar{\Gamma}_{1}=\bar{\Gamma}_{1}(x ; \bar{p}), \quad \bar{\Gamma}_{2}=\bar{\Gamma}_{2}(x ; \bar{p})$, is not linear integral in the $\bar{p} s$. Also no one of these is a non-zero constant multiple of any other.

By Theorem 20.1, these three distinct infinitesimal contact transformations $U F, \bar{U}_{1} F, \bar{U}_{2} F$, possess the same transversality law if and only 
if the three associated characteristic functions $\mathrm{\Gamma}=\mathrm{\Gamma}(x ; \bar{p}), \bar{\Gamma}_{1}=\bar{\Gamma}_{1}(x ; \bar{p})$, $\bar{\Gamma}_{2}=\bar{\Gamma}_{2}(x ; \bar{p})$, are related by two equations of the form

$$
\bar{\Gamma}_{1}=\bar{\Gamma}_{1}(x ; \bar{p})=\frac{1}{\mu_{1}(x)} \Gamma(x ; \bar{p}), \overline{\Gamma_{2}}=\bar{\Gamma}_{2}(x ; \bar{p})=\frac{1}{\mu_{2}(x)} \Gamma(x ; \bar{p}),
$$

where each one of the two point functions $\mu_{1}(x)$ and $\mu_{2}(x)$, is at least of class two and never vanishes in a certain $n$ dimensional region of points $x$. Furthermore, neither one of these two point functions $\mu_{1}(x)$ and $\mu_{2}(x)$, is a non-zero constant multiple of the other.

THEOREM 20.2 - The characteristic function $\bar{\Gamma}_{3}=\bar{\Gamma}_{3}(x ; \bar{p})$, of the commutator $\left({\overline{U_{1}}}_{1},{\overline{U_{2}}}_{2}\right) F$ of the two distinct infinitesimal contact transformations $\bar{U}_{1} F$ and $\bar{U}_{2} F$ for which the two characteristic functions are $\bar{\Gamma}_{1}=\bar{\Gamma}_{1}(x ; \bar{p})=$ $=\frac{1}{\mu_{1}(x)} \Gamma(x ; \bar{p})$, and $\bar{\Gamma}_{2}=\bar{\Gamma}_{2}(x ; \bar{p})=\frac{1}{\mu_{2}(x)} \Gamma(x ; \bar{p})$, is

$$
\bar{\Gamma}_{3}=\left(\bar{\Gamma}_{1}, \bar{\Gamma}_{2}\right)=\frac{1}{2 \mu_{1}^{2} \mu_{2}^{2}}\left(\mu_{2} \frac{\partial \mu_{1}}{\partial x^{\alpha}}-\mu_{1} \frac{\partial \mu_{2}}{\partial x^{\alpha}}\right) \frac{\partial}{\partial p_{\alpha}}\left(\Gamma^{2}\right)
$$

This follows by substituting (20.6) into (20,2).

The remaining theorems of this section are extensions of some theorems of KASNER concerning the commutator $\left(\bar{U}_{1}, \bar{U}_{2}\right) F$ of two distinct infinitesimal contact transformations $\bar{U}_{1} F$ and $\vec{U}_{2} F$ associated with two distinet dynamical families.

The characteristic function $\mathrm{\Gamma}=\Gamma(x ; p)$, of a given Riemannian space $V_{n}$, is $\Gamma=\Gamma(x ; p)=\left(g^{\alpha \beta} p_{\alpha} p_{\beta}\right) \frac{1}{2}$ For this Riemannian space $V_{n}$, the transversality law is that of orthogonality.

In the Riemannian space $\nabla_{n}$, a conservative physical family $S_{k}(E)$ where $k \neq-1, \infty$, of $\infty^{2 n-2}$ trajectories $C$, or a conservative velocity family $S_{\infty}\left(v_{0}\right)$ of $\infty^{2 n-2}$ velocity curves $C$, is a natural family $F$ of $\infty^{2 n-2}$ curves $C$, and conversely. The characteristic function $\bar{\Gamma}=\bar{\Gamma}(x ; \bar{p})$, of the associated infinitesimal contact transformation $\bar{U} F$, of this natural family $F$ of $\infty^{2 n-2}$ curves $C$, is

$$
\bar{\Gamma}=\bar{\Gamma}(x ; \bar{p})=\frac{1}{\mu(x)} \Gamma(x ; \bar{p})=\frac{1}{\mu(x)}\left(g^{\alpha \beta} \bar{p}_{x} \bar{p}_{\beta}\right)^{\frac{1}{2}}
$$

Thus the transversality law of the infinitesimal contact transformation $\bar{U} F$, is that of orthogonality in the Riemannian space $V_{n}$.

THEOREM 20.3. - The commutator $\left(\bar{U}_{1}, \bar{U}_{2}\right) F$ of two distinct infinitesimal contact transformation $\bar{U}_{1} F$ and $\bar{U}_{2} F$ of two distinct natural families $F_{1}$ and $\mathrm{F}_{2}$ of the same Riemannian space $\nabla_{n}$, is a point transformation. 
For, by hypothesis, it is seen that $\Gamma^{2}=g^{\alpha} \bar{p}_{x} \bar{p}_{\beta}$. Then by (20.7) and (20.8), the characteristic funcion $\bar{\Gamma}_{3}=\bar{\Gamma}_{3}(x ; \bar{p})$ of the commutator $\left(\bar{U}_{1}, \bar{U}_{2}\right) F$, is

$$
\bar{\Gamma}_{3}=\bar{\Gamma}_{3}(x ; \bar{p})=\frac{1}{\mu_{1}^{2} \mu_{2}^{2}}\left(\mu_{2} \frac{\partial \mu_{1}}{\partial x^{\alpha}}-\mu_{1} \frac{\partial \mu_{2}}{\partial x^{\alpha}}\right)\left(g^{x \beta} \bar{p}_{\beta}\right)
$$

Since $\bar{\Gamma}_{3}=\bar{\Gamma}_{3}(x ; \bar{p})$, is linear integral in the $\overline{p^{\prime}} s$, it follows that $\left(\bar{U}_{1}, \bar{U}_{2}\right) F$, is a point transformation.

THЕоRем 20.4 - If two distinct infinitesimal contact transformations $\bar{U}_{1} F$ and $\bar{U}_{2} F$, possess the same linear involutorial law of transversals, then the commutator $\left(\bar{U}_{1}, \bar{U}_{2}\right) F$, is a point correspondence.

This is a restatement of Theorem 20.3 .

THEOREM 20.5 - The commutator $\left.\bar{U}_{1}, \bar{U}_{2}\right) F$ of two distinct infinitesimal contact transformations $\bar{U}_{1} F$ and $\bar{\Pi}_{2} F$ of two distinct natural families $F_{1}$ and $F_{2}$ of two Riemannian spaces $V_{n}$ and $\bar{V}_{n}$, is a point correspondence $T$ if and only if $V_{n}$ and $\bar{V}_{n}$ are conformally equivalent.

For, there is no loss in generality in assuming that the two characteristic functions $\bar{\Gamma}_{1}=\bar{\Gamma}_{1}(x ; \bar{p})$ and $\bar{\Gamma}_{2}=\bar{\Gamma}_{2}(x ; \bar{p})$, of the two distinct infinitesimal contact tranformations $\bar{U}_{1} F$ and $\bar{U}_{2} F$, be of the forms

$$
\bar{\Gamma}_{1}=\bar{\Gamma}_{1}(x ; \bar{p})=\left(g^{\alpha \beta} \bar{p}_{\alpha} \bar{p}_{\beta}\right)^{1 / 2}, \bar{\Gamma}_{2}=\bar{\Gamma}_{2}(x ; \bar{p})=\left(\bar{g}^{\alpha \beta} \bar{p}_{\alpha} \bar{p}_{\beta}\right)^{1 / 2}
$$

for which the two quadratic forms $g^{\alpha \mathrm{P}} \bar{p}_{\alpha} \bar{p}_{\beta}$, and $\bar{g}^{\alpha \beta} \bar{p}_{\alpha} \bar{p}_{\beta}$, are positive definite. By (20.2), the characteristic function $\bar{\Gamma}_{3}=\bar{\Gamma}_{3}(x ; \bar{p})$ of the commutator $\left(\bar{U}_{1}, \bar{U}_{2}\right) F$, is

$$
\bar{\Gamma}_{3}=\bar{\Gamma}_{3}(x ; \bar{p})=\frac{1}{2 \bar{\Gamma}_{1} \bar{\Gamma}_{2}}\left(g^{i \alpha} \frac{\partial \bar{g}^{j k}}{\partial x^{\alpha}}-\bar{g}^{i \alpha} \frac{\partial g^{j k}}{\partial x^{\alpha}}\right) \bar{p}_{i} \bar{p}_{j} \bar{p}_{k}
$$

This is the characteristic function $\bar{\Gamma}_{3}=\bar{\Gamma}_{3}(x ; \bar{p})$ of a point transformation $\left(\bar{U}_{1}, \bar{U}_{2}\right) F$, if and only if $\bar{\Gamma}_{3}=\bar{\Gamma}_{3}(x ; \bar{p})$, is linear integral in the $\bar{p}^{\prime} s$. Under these conditions, there exists a positive scalar point function $\mu(x)>0$, such that $\bar{g}^{i j}=\frac{1}{\mu^{2}} g^{i j}$. This means that the two Riemannian spaces $V_{n}$ and $\bar{V}_{n}$ are conformally equivalent.

Theorem 20.6 - Two distinct infinitesimal contact transformations $\bar{U}_{1} F$ and $\bar{U}_{2} F$ with the same transversality law, possess a point transformation $T$ 
for their commutator $\left(\bar{U}_{1}, \bar{U}_{2}\right) F$, if and only if they are associated with two distinct natural families $F_{1}$ and $F_{2}$ of two conformally equivalent Riemannian spaces $V_{n}$ and $\bar{V}_{n}$.

For, under the given hypotheses, it follows by Theorem 20.1, that the two characteristic functions $\bar{\Gamma}_{1}=\bar{\Gamma}_{1}(x ; \bar{p})$, and $\bar{\Gamma}_{2}=\bar{\Gamma}_{2}(x ; \bar{p})$, of the two distinct infinitesimal transformations $\bar{U}_{1} F$ and $\bar{U}_{2} F$, can be taken to be

$$
\bar{\Gamma}_{1}=\bar{\Gamma}_{1}(x ; \bar{p})=\Gamma(x ; \bar{p}), \bar{\Gamma}_{2}=\bar{\Gamma}_{2}(x ; \bar{p})=\lambda(x) \Gamma(x ; \bar{p})
$$

where $\lambda(x)$ is a point function which is not a constant in the given $n$ dimensional region of points $x$, The characteristic function $\bar{\Gamma}_{3}=\bar{\Gamma}_{3}(x ; \bar{p})$ of the commutator $\left(\bar{U}_{1}, \bar{U}_{2}\right) F$, is

$$
\overline{\Gamma_{3}}=\overline{\Gamma_{3}}(x ; \bar{p})=\frac{1}{2} \frac{\partial \lambda}{\partial x^{\alpha}} \frac{\partial}{\partial \bar{p}_{\alpha}} \Gamma^{2}
$$

Thus the commutator $\left(\bar{U}_{1}, \bar{U}_{2}\right) F$, is a point transformation $T$ if and only if

$$
\frac{\partial \lambda}{\partial x^{\alpha}} \frac{\partial^{3} \Gamma^{2}}{\partial \bar{p}_{x} \partial \bar{p}_{\beta} \partial \bar{p}_{Y}}=0
$$

Suppose that at least one of the third order partial derivatives $\partial^{3} \Gamma^{2} / \partial \bar{p}_{x} \partial \bar{p}_{\beta} \partial \bar{p}_{Y}$, is not zero. Then (20.14), is a linear homogeneous partial differential equation of first order in the function $\lambda$. By standard theory, this possesses $(2 n-1)$ functionally independent solutions, and any other solution is a function of these. Now evidently, $\left(\bar{p}_{1}, \ldots, \bar{p}_{n}\right)$, are $n$ functionally independent solutions. Hence there exist $(n-1)$ functionally independent solutions $\lambda_{1}(x), \ldots, \lambda_{n-1}(x)$, depending on the points $x$, only. Replacing $\lambda$ in (20.14) by each one of these $(n-1)$ expressions $\lambda_{1}(x), \ldots, \lambda_{n-1}(x)$, it follows that the quantities $\partial^{3} \Gamma^{2} / \partial \bar{p}_{\alpha} \partial \bar{p}_{\rho} \partial \bar{p}_{\gamma}$, are proportional to functions of the points $x$ only.

But $\partial^{2} \Gamma^{2} / \partial \bar{p}_{\beta} \partial \bar{p}_{\gamma}$, is homogeneous of degree zero in the $\overline{p^{\prime}} s$. Thus by Euler's theorem on homogeneous functions, it follows that

$$
\bar{p}_{\alpha} \frac{\partial^{3} \Gamma^{2}}{\partial \bar{p}_{\alpha}{ }^{2} \bar{p}_{\beta} \partial \bar{p}_{Y}}=0
$$

Hence since the third order partial derivatives of $\Gamma^{2}$ with respect to 
the $\bar{p}_{s^{t}}$, are proportional to functions of the points $x$ only, it follows that they must vanish identically.

This is a contradiction of our supposition.

By the preceding argument, it is seen that

$$
\frac{\partial^{3} \Gamma^{2}}{\partial \bar{p}_{\alpha} \partial \bar{p}_{\beta} \partial p_{\gamma}}=0, \text { for } \partial, \beta, \gamma=1,2, \ldots, n \text {. }
$$

Therefore, $\Gamma^{2}$ is a homogeneous quadratic form in the $\bar{p}^{\prime} s$. Moreover, $\Gamma^{2}$ is a positive definite quadratic form in the $\bar{p}^{\prime} s$.

Substituting the positive definite quadratic form $\Gamma^{2}$ in the $\overline{p^{\prime}} s$, into (20.12), it is seen that the proof of Theorem 20.6, is complete.

21. Geodesically equivalent Riemannian spaces $V_{n}$ and $\bar{V}_{n}[17]$. A projective transformation $T$ between two Riemannian spaces $V_{n}$ and $\bar{V}_{n}$, is a cartogram $T$ or a correspondence $T$ between $V_{n}$ and $\bar{V}_{n}$, such that under $T$, every geodesic $C$ of $V_{n}$, is converted into a geodesic $\bar{C}$ of $\bar{V}_{n}$. Any two such Riemannian spaces $V_{n}$ and $\bar{V}_{n}$ are said to be geodesically equivalent.

Theorem 21.1 - A correspondence $T$ between two Riemannian spaces $V_{n}$ and $\bar{V}_{n}$, is a projective transformation $T$ if and only if the Christoffel symbols of the second kind: $\Gamma_{j k}^{i}$ and $\overline{\mathbf{\Gamma}}_{j k}^{i}$ of $V_{n}$ and $\bar{V}_{n}$, are related as follows

$$
\begin{aligned}
& \bar{\Gamma}_{j k}^{i}=\Gamma_{j k}^{i}, \text { for } j \neq i, \text { and } k \neq i \\
& \bar{\Gamma}_{i i}^{i}-2 \bar{\Gamma}_{i j}^{j}=\Gamma_{i i}^{i}-2 \Gamma_{i j}^{j}, \text { for } j \neq i .
\end{aligned}
$$

This system of equations is equivalent to the set of equations: $\bar{\Delta}^{i}=\Delta^{i}$, for $i=1,2, \ldots, n$.

For, by equation (7.1), the correspondence $T$ is a projective transformation $T$ between the two Riemannian spaces $V_{n}$ and $\bar{V}_{n}$, if and only if $\bar{\Delta}^{i}=\Delta^{i}$, for $i=1,2, \ldots, n$. From (7.2), this set of equations, namely $\bar{\Delta}^{i}=\Delta^{i}$, for $i=1,2, \ldots, n$, is equivalent to the system of equations

$$
\begin{aligned}
& \bar{\Gamma}_{j j}^{i}=\Gamma_{j j}^{i}, \quad \bar{\Gamma}_{j j}^{j}-2 \bar{\Gamma}_{i j}^{i}=\Gamma_{j j}^{j}-2 \Gamma_{i j}^{i}, \bar{\Gamma}_{\alpha j}^{i}=\Gamma_{\alpha j}^{i}, \\
& \bar{\Gamma}_{i i}^{i}-2 \bar{\Gamma}_{i j}^{j}=\Gamma_{i j}^{i}-2 \Gamma_{i j}^{j}, \quad \bar{\Gamma}_{\alpha j}^{j}-\Gamma_{\alpha i}^{i}=\Gamma_{\alpha j}^{j}-\Gamma_{\alpha i}^{i}, \\
& \bar{\Gamma}_{i i}^{j}=\Gamma_{i i}^{i}, \quad \bar{\Gamma}_{\alpha i}^{j}=\Gamma_{\alpha i}^{j}, \quad \bar{\Gamma}_{\alpha \beta}^{j}=\Gamma_{\alpha \beta}^{j},
\end{aligned}
$$


for $i \neq j ; \alpha \neq i, j ; \beta \neq i, j$. This system (21.2), is equivalent to the system (21.1). Thus Theorem 21.1, is proved.

If the two Riemannian space $V_{n}$ and $\bar{V}_{n}$ are not geodesically equivalent under a given cartogram $T$, then all the possible geodesic $C$ of $V_{n}$, which by $T$, are converted into geodesic $\bar{C}$ of $\bar{V}_{n}$, are integral solutions $C$ of $(n-1)$ explicit cubic differential equations, each of the first order. If these $(n-1)$ cubic dfferential equations are all independent of one another, the maximum number of geodesic $O$ of $V_{n}$ so converted, is $3^{n-1} \infty^{n-1}$. This extends to $n$ dimensions, a corresponding theorem of KasNer for surfaces.

An affine transtormation $T$ between two Riemannian spaces $V_{n}$ and $\bar{V}_{n}$, is a cartogram $T$ or a correspondence $T$, such that under $T$, the CHRISToFfeL symbols of the second kind: $\Gamma_{j k}^{i}$ and $\Gamma_{j k}^{i}$, of $V_{n}$ and $\bar{V}_{n}$, are invariant. That is, $\bar{\Gamma}_{j k}^{i}=\Gamma_{j k}^{i}$, for $i, j, k=1,2, \ldots, n$. Then the two Riemannian space $V_{n}$ and $\bar{V}_{n}$ are said to be affinely equivalent.

An affine transformation $T$ between two Riemannian spaces $V_{n}$ and $\bar{V}_{n}$, is a projective transformation $T$. Hence if two Riemannian spaces $V_{n}$ and $\bar{V}_{n}$, are affinely equivalent, then they are geodesically equivalent.

However, the converse of the above statement is not always true. That is, there may exist a projective transformation $T$ between two Riemannian spaces $V_{n}$ and $\vec{V}_{n}$ which is not an affine correspondence $T$.

It is clear that an affine transformation $T$ between two Riemannian spaces $V_{n}$ and $\bar{V}_{n}$, preserves parallel displacements of vectors.

TheoRem 21.2. - If $T$ is both a conformal map and a projective transformation between two Riemannian spaces $V_{n}$ and $\bar{\nabla}_{n}$, then $T$ is a homothetic correspondence, and $\bar{V}_{n}$ is a homothetic representation of $\nabla_{n}$. That is, ds $=\mu d s$, where $\mu>0$, is a constant.

For, let $T$ denote a conformal cartogram $T$ between two Riemannian spaces $V_{n}$ and $\bar{V}_{n}$. Then $d \bar{s}=\mu d s$, where $\mu>0$, is a positive scalar point function. Thus $\bar{g}_{i j}=\mu^{2} g_{i j}$, and $\bar{g}^{i j}=\frac{1}{\mu^{2}} g^{i j}$. By $(10.3)$, it is seen that

$$
\bar{\Gamma}_{j k}^{i}=1_{j k}^{i}+\frac{1}{\mu}\left(\delta_{j}^{i} \frac{\partial \mu}{\partial x^{k}}+\delta_{k}^{i} \frac{\partial \mu}{\partial x^{j}}-g_{j k} g^{i l} \frac{\partial \mu}{\partial x^{l}}\right)
$$

If this conformal cartogram $T$ is also a projective transformation $T$, then necessarily, $\bar{\Gamma}_{j k}^{i}=\Gamma_{i k}^{i}$, for $j \neq i$, and $k \neq i$. Thus a necessary set of conditions for this, is

$$
g_{j k} g^{i l} \frac{\partial \mu}{\partial x^{l}}=0
$$

for $j \neq i$, and $k \neq i$. 
J. De Cicco: The Riemannian Geometry of Physical Systems, etc. 395

If $g_{s}=0$, for all $j \neq i$, and all $k \neq i$, then since $i$ is a random index, it follows that $g_{i j}=0$ for all $i, j=1,2, \ldots, n$. Hence there is at least one $g_{j k} \neq 0$, for $j \neq \mathrm{i}$, and $k \neq i$.

For this particular $g_{j k} \neq 0$, for $j \neq i$, and $k \neq i$, it follows from (21.4) that

$$
g^{i l} \frac{\partial \mu}{\partial x^{i}}=0
$$

for $i=1,2, \ldots, n$. As the determinant $\left|g^{i l}\right|=\frac{1}{g} \neq 0$, it is seen that $\frac{\partial \mu}{\partial x^{l}}=0$, for $l=1,2, \ldots, n$. Therefore $\mu>0$, is a positive constant.

As it is evident that the necessary condition $d \bar{s}=\mu d s$, where $\mu>0$, is a positive constant, is also a sufficient condition, the Theorem 21.2, is established.

From (21.3), it is seen that a homothetic transformation $T$ between two Riemannian spaces $V_{n}$ and $\bar{V}_{n}$, is an affine transformation $T$. Therefore, if two Riemannian spaces $V_{n}$ and $\bar{V}_{n}$, are homothetically equivalent, then they are affinely equivalent.

The converse of the above result is not always true. Thus, there may exist an affine transformation $T$ between two Riemannian spaces $V_{n}$ and $\bar{V}_{n}$ which is not a homothetic map $T$.

22. Appell's transformation $T$ for a dynamical system $S_{0}$ of $\infty^{2 n-1}$ dynamical trajectories $C$, in a Riemannian space $\nabla_{n}$ [18]. From Theorem 7.1, the explicit differential equations of a complete dynamical system $S_{0}$ of $\infty^{2 n-1}$ dynamical. trajectories $C$, in a Rieniannian space $V_{n}$, are

$$
+\left[\begin{array}{c}
\frac{d \Phi^{i}}{d x^{j}}-\frac{d \Phi^{j}}{d x^{j}} \frac{d x^{i}}{d x^{j}}+\Phi^{i}\left(\Gamma_{\alpha \beta}^{i} \frac{d x^{\alpha}}{d x^{j}} \frac{d x^{\beta}}{d x^{j}}-\Gamma_{\alpha \beta}^{j} \frac{d x^{\alpha}}{d x^{j}} \frac{d x^{\beta}}{d x^{i}} \frac{d x^{i}}{d x^{j}}\right) \\
+2 \Gamma_{\alpha \beta}^{j} \frac{d x^{\alpha}}{d x^{j}} \frac{d x^{\beta}}{d x^{j}}\left(\Phi^{i}-\Phi^{j} \frac{d x^{i}}{d x^{j}}\right)
\end{array}\right] \Delta^{i}
$$

where the two indices $i$ and $j$ with $i \neq j$, are held fixed, and $l \neq i$, is allowed to vary over the remaining integers 1 to $n$. 
TheoRem 22.1. - Appell's Transformation T For A Kiemannian Space $V_{n}$. $A$ transformation $T$ between two Riemannian spaces $V_{n}$ and $\vec{V}_{n}$, converts every complete dynamical system $S_{0}$ of $\infty^{2 n-1}$ dynamical trajectories $C$ in $V_{n}$, into a complete dynamical system $\bar{S}_{0}$ of $\infty^{2 n-1}$ dynanical trajectories $\bar{C}$ in $\bar{V}_{n}$, if and only if it is a projective transformation $T$ between the two Riemannian spaces $V_{n}$ and $V_{n}$, such that the following set of $\frac{n}{2}(n-1)$ conditions, os valid, namely

$$
\frac{\partial}{\partial x^{j}}\left(\bar{\Gamma}_{i i}^{i}-\Gamma_{i i}^{i}\right)=\frac{\partial}{\partial x^{i}}\left(\bar{\Gamma}_{j i}^{j}-\Gamma_{j j}^{j}\right),
$$

for $i \neq j$, and $i, j,=1,2, \ldots, n$. Movorover, the two contravariant torms $\bar{\Phi}^{i}$ and $\Phi^{i}$ of the two positional fields of force $\bar{\Phi}$ and $\Phi$, satisfy the $n$ conditions

$$
\Phi^{i}= \pm \mu^{2} \Phi^{i}
$$

for $i=1,2, \ldots, n$, where $\mu=\mu(x)>0$, is a positive scalar point function of at least class two in a certain $n$ dimensional region of points $x$ for which

$$
-\frac{1}{\mu} \frac{\partial \mu}{\partial x^{i}}=\bar{\Gamma}_{i i}^{i}-\Gamma_{i i}^{i}
$$

for $i=1,2, \ldots, n$. Any such correspondence is called Appell's transfor. mation $T$.

For, let $T$ be any point correspondence between two Riemannian spaces $V_{n}$ and $\bar{V}_{n}$, which carries every complete dynamical system $S_{0}$ of $\infty^{2 n-1}$ dynamical trajectories $C$ in $V_{n}$ into a complete dynamical system $\bar{S}_{0}$ of $\infty^{2 n-1}$ dynamical trajectories $\bar{C}$ in $\bar{V}_{n}$.

Now the logical intersection of all complete dynamical systems $S_{0}$ in $V_{n}$, is the family $F$ of $\infty^{2 n-2}$ geodesies $C$ in $V_{n}$. Similarly, the logical intersection of all complete dynamical systems $\bar{S}_{0}$ in $\bar{V}_{n}$, is the family $\bar{F}$ of $\infty^{2 n-2}$ geodesics $\bar{C}$ in $\bar{V}_{n}$.

By these remarks, the required map $T$ converts the family $F$ of $\infty^{2 n-2}$ geodesics $C$ in $V_{n}$, into the family $\bar{F}$ of $\infty^{2 n-2}$ geodesics $\bar{C}$ in $\bar{V}_{n}$. Consequently, it is necessarily a projective transformation $T$ between the two Riemannian spaces $V_{n}$ and $\bar{V}_{n}$.

For this projective transformation $T$, the set of conditions (21.1), is fulfulled. Thus $\bar{\Delta}^{i}=\Delta^{i}$, and $\frac{d \bar{\Delta}^{i}}{d x^{j}}=\frac{d \Delta^{i}}{d x^{j}}$, for all $i, j=1,2, \ldots, n$.

Because of the preceding conditions and because of equations (22.1), the two corresponding positional fields of force $\Phi$ and $\bar{\Phi}$, are related by 
the conditions (22.3). If the plus sign is chosen in (22.3), the actual and virtual dynamical trajectories $C$ of the complete dynamical system $S_{0}$ in $V_{n}$, correspond to the actual and virtual dynamical trajectories $\bar{C}$ of the complete dynamical system $\bar{S}_{0}$ in $\bar{V}_{n}$. On the other hand, if the minus sign is chosen in (22.3), the actual and virtual dynamical trajectories $C$ of the complete dynamical system $S_{0}$ in $V_{n}$, correspond to the virtual and actual dynamical trajectories $\bar{C}$ of the complete dynamical system $\bar{S}_{0}$ in $\bar{V}_{n}$.

By equations (22.1) and (22.3), the only remaining conditions that the required projective transformation $T$ must fulfill, are

$$
\begin{aligned}
& \frac{d \bar{\Phi}^{i}}{d x^{j}}-\frac{d \bar{\Phi}^{i}}{d x^{j}} \frac{d x^{i}}{d x^{j}} \\
& \bar{\Phi}^{i}-\bar{\Phi}^{j} \frac{d x^{i}}{d x^{j}}+2 \bar{\Gamma}_{\alpha \beta}^{i} \frac{d x^{\alpha}}{d x^{j}} \frac{d x^{\beta}}{d x^{j}}+\frac{\bar{\Phi}^{j}}{\bar{\Phi}^{i}-\bar{\Phi}^{j} \frac{d x^{i}}{d x^{j}}}\left[\bar{\Delta}^{i}-\frac{d^{2} x^{i}}{d\left(x^{j}\right)^{2}}\right] \\
&=\frac{\frac{d \Phi^{i}}{d x^{j}}-\frac{d \Phi^{i}}{d x^{i}} \frac{d x^{i}}{d y^{j}}}{\Phi^{i}-\Phi^{j} \frac{d x^{i}}{d x^{j}}}+2 \Gamma_{\alpha \beta}^{j} \frac{d x^{\alpha}}{d x^{j}} \frac{d x^{\beta}}{d x^{j}}+\frac{\Phi^{i}}{\Phi^{i}-\Phi^{j} \frac{d x^{i}}{d x^{j}}}\left[\Delta^{i}-\frac{d^{2} x^{i}}{d\left(x^{j}\right)^{2}}\right],
\end{aligned}
$$

with $i \neq j$, and $i, j=1,2, \ldots$. Substituting $\bar{\Phi}^{i}= \pm \mu^{2} \Phi^{i}$, these become the single condition

$$
\frac{1}{\mu} \frac{d \mu}{d x^{i}}+\left(\bar{\Gamma}_{\alpha \beta}^{i}-\Gamma_{\alpha \beta}^{j}\right) \frac{d x^{\alpha}}{d x^{j}} \frac{d x^{\beta}}{d x^{j}}=O
$$

By use of equations (21.1), this becomes

$$
\frac{1}{\mu} \frac{d \mu}{d x^{j}}+\left(\bar{\Gamma}_{j j}^{j}-\Gamma_{j j}^{j}\right)+\underset{\alpha \neq j}{\Sigma}\left(\bar{\Gamma}_{\alpha \alpha}^{\alpha}-\Gamma_{\alpha \alpha}^{\alpha}\right) \frac{d x^{\alpha}}{d x^{j}}=0
$$

This is the same as saying that

$$
-\frac{1}{\mu} d \mu=\left(\bar{\Gamma}_{i i}^{i}-\Gamma_{i i}^{i}\right) d x^{i}
$$

The conditions (22.2) are those for the exact integrability of the Pfaffian that appears on the right of equation (22.8). Finally the conditions (22.4), are obtained from this equation (22.8) by equating the corresponding coefficients of $d x^{i}$. 
Therefore a projective transformation $T$ between two Riemannian spaces $V_{n}$ and $\bar{V}_{n}$, is an Appell transformation $T$ if and only if it obeys the set of $\frac{n}{2}(n-1)$ conditions (22.2).

This completes the proof of Theorem 22.1.

Theorem 22.2 - Under a point correspondence T between two Riemannian spaces $V_{n}$ and $\bar{V}_{n}$, consider a change of the times $t$ and $\bar{t}$ of the form

$$
d \bar{t}=\frac{1}{\mu} d t
$$

where $\mu=\mu(x)>0$, is a positive scalar point function $\mu=\mu(x)>0$, of at least class two in a cerlain $n$ dimensional region of points $x$. This is called an associated positive scalar time point function $\mu=\mu(x)>0$. The contravariant forms $v^{t}$ and $\overline{v^{t}}$ of the two corresponding velocity vectors $v$ and $\bar{v}$, are given by the set of $n$ conditions

$$
\bar{v}^{i}=\mu v^{i}
$$

for $i=1,2, \ldots, n$. The contravariant forms $a^{i}$ and $\bar{a}^{i}$ of the two corre. sponding acceleration vectors $a$ and $\bar{a}$, obey the set of $n$ relations

$$
\bar{a}^{i}=\mu^{2} a^{i}+\mu^{2}\left[\frac{1}{\mu} \frac{d \mu}{d t} \frac{d x^{i}}{d t}+\left(\bar{\Gamma}_{j k}^{i}-\Gamma_{j k}^{i}\right) \frac{d x^{j}}{d t} \frac{d x^{k}}{d t}\right]
$$

for $i=1,2, \ldots, n$. A point correspondence $T$ is an Appell transformation $T$ between the two Riemannian spaces $V_{n}$ and $\bar{V}_{n}$ if and only if

$$
\bar{a}^{i}=\mu^{2} \alpha^{i}
$$

for $i=1,2, \ldots, n$.

For, by (22.9), $\frac{d x^{i}}{d \bar{t}}=\mu \frac{d x^{i}}{d t}$, and hence the equations (22.10), are obtained.

By (22.9), it is seen that

$$
\frac{d x^{i}}{d \bar{t}}=\mu \frac{d x^{i}}{d t}, \frac{d^{2} x^{i}}{d \bar{t}^{2}}=\mu^{2} \frac{d^{2} x^{i}}{d t^{2}}+\mu \frac{d \mu}{d t} \frac{d x^{i}}{d t}
$$


Then

$$
\begin{aligned}
& \vec{a}^{i}=\frac{d^{2} x^{i}}{d \bar{t}^{2}}+\bar{\Gamma}_{j k}^{i} \frac{d x^{j}}{d \bar{t}} \frac{d x^{k}}{d \bar{t}}=\mu^{2} \frac{d^{2} x^{i}}{d t^{2}}+\mu \frac{d \mu}{d t} \frac{d x^{i}}{d t}+\mu^{2} \bar{\Gamma}_{j k}^{i} \frac{d x^{j}}{d t} \frac{d x^{k}}{d t} \\
& =\mu^{2} a^{i}+\mu^{2}\left[\frac{1}{\mu} \frac{d \mu}{d t} \frac{d x^{i}}{d t}+\left(\bar{\Gamma}_{j k}^{i}-\Gamma_{j k}^{i}\right) \frac{d x^{j}}{d t} \frac{d x^{k}}{d t}\right] .
\end{aligned}
$$

Thus the equations (22.11) have been derived.

The equations (22.11) may be written in the form

$$
\begin{aligned}
& \frac{1}{\mu^{2}}\left(\bar{a}^{i}-\mu^{2} a^{i}\right)=\left(\frac{1}{\mu} \frac{\partial \mu}{\partial x^{i}}+\bar{\Gamma}_{i i}^{i}-\Gamma_{i i}^{i}\right) \frac{d x^{i}}{d t} \frac{d x^{i}}{d t} \\
& +\sum_{j \neq i}\left(\frac{1}{\mu} \frac{\partial \mu}{\partial x^{j}}+2 \bar{\Gamma}_{i j}^{i}-2 \Gamma_{i j}^{i}\right) \frac{d x^{i}}{d t} \frac{d x^{j}}{d t}+\sum_{\substack{j \neq i \\
k \neq i}}\left(\bar{\Gamma}_{j k}^{i}-\Gamma_{j k}^{i}\right) \frac{d x^{j}}{d t} \frac{d x^{k}}{d t} .
\end{aligned}
$$

From these equations, it is seen that the conditions $a^{i}=\mu^{2} a^{i}$, hold identically, if and only if

$$
\begin{aligned}
& -\frac{1}{\mu} \frac{\partial \mu}{\partial x^{i}}=\bar{\Gamma}_{i i}^{i}-\Gamma_{i i}^{i}, \quad \bar{\Gamma}_{i i}^{i}-2 \bar{\Gamma}_{i j}^{j}=\Gamma_{i i}^{i}-2 \Gamma_{i j}^{j}, \text { for } j \neq i, \\
& \bar{\Gamma}_{j k}^{i}=\Gamma_{j k}^{i}, \text { for } j \neq i, \quad k \neq i .
\end{aligned}
$$

Therefore the point correspondence $T$ is an APPELL transformation $T$ between the two Riemannian spaces $V_{n}$ and $\vec{V}_{n}$, and conversely.

Consequently the proof of Theorem 22.2, has been completed.

It is remarked that if an associated positive scalar time point function of an APPELL transformation $T$, is $\mu=\mu(x)>0$, then any other associated positive scalar time point function of the same APPELL transformation $T$, is $\nu=a \mu>0$, where $a>0$, is an arbitrary positive multplicative constant.

THEOREM 22.3 - A point correspondence $T$ is an affine transformation $T$ between two Riemannian spaces $\nabla_{n}$ and $\bar{V}_{n}$, and only if it converts every complete dynamical system $S_{0}$ of $\infty^{2 n-1}$ dynamical trajectories $C$ in $\nabla_{n}$, into a complete dynamical system $\bar{S}_{0}$ of $\infty^{2 n-1}$ dynamical trajectories $\bar{C}$ in $\bar{V}_{n}$, such that an associated positive scalar time point function $\mu>0$, is a constant.

For, such a point correspondence $T$ is necessarily an APPELL transformation $T$. Since $\mu>0$, is constant, it follows that $\bar{\Gamma}_{i i}^{i}=\Gamma_{i i}^{i}$, by (22.4). From equations (21.1), it is deduced that $\bar{\Gamma}_{j k}^{i}=\Gamma_{j k}^{i}$, for all $i, j, k=1,2, \ldots, n$. 
Thus the required point correspondence $T$ is an affine transformation $T$ between the two Riemannian spaces $V_{n}$ and $\bar{V}_{n}$.

Let $x^{i}$ and $\bar{x}^{i}$ denote cartesian coordinates of points $x$ and $\bar{x}$ in two Euclidean spaces $V_{n}$ and $\bar{\nabla}_{n}$. Then AppecL's transformation $T$ is

$$
\bar{x}^{i}=\frac{a_{j}^{i} x^{i}+b^{i}}{a_{j}^{0} x^{i}+b^{0}}, \quad d t=\frac{d t}{k^{2}\left(a_{j}^{0} x^{j}+b^{0}\right)^{2}},
$$

for $i=1,2, \ldots, n$, where $k>0$, is a constant, and the matrix $\left(a_{j}^{i} ; b^{i}\right)$ for $i=0,1,2, \ldots, n$, and $j=1,2, \ldots n$, is of rank $(n+1)$.

Here the associated positive scalar time point function $\mu>0$, is

$$
\mu=k^{2}\left(a_{j}^{0} x^{i}+b^{0}\right)^{2}>0
$$

It is a constant if and only if $a_{j}^{0}=0$ for $j=1,2, \ldots, n$, and $b^{0} \neq 0$. Then $(22.17)$, is an affine transformation $T$.

23. Transformation theory of physical systems $S_{k}$ where $k \neq-\mathbf{1 , 0}$, but where $k$ may be infinite, of $\infty^{2 n-1}$ trajectories $C$, in a Riemannian space $V_{n}$ [19]. Consider a point correspondence $T$ which carries every complete physical system $S_{k}$ with $k \neq \ldots 1$, 0, of $\infty^{2 n-1}$ trajectories $C$ of a Riemannian space $V_{n}$, into a complete physical system $\bar{S}_{k}$ with the same $k \neq-1,0$, of $\infty^{3 n-1}$ trajectories $\bar{C}$ of a Riemannian space $\bar{V}_{n}$. Here $k$ may be infinite in which case, the transformation theory of velocity systems $S_{\infty}$ in included as a special case. Of course, $k \neq 0$, since the transformation theory of dynamical systems $S_{0}$, has already been considered.

By an examination of equations (7.9), such a correspondence $T$ is necessarily a projectice transformation $T$ between the two Riemannian spaces $\nabla_{n}$ and $\bar{\nabla}_{n}$.

If $n \geqq 3$, then under $T$, the following set of $(n-2)$ identities must be satisfied, namely

$$
\frac{\bar{\Phi}^{l} d x^{j}-\bar{\Phi}^{j} d x^{l}}{\bar{\Phi}^{i} d x^{j}-\bar{\Phi}^{j} d x^{i}}=\frac{\Phi^{l} d x^{j}-\Phi^{j} d x^{l}}{\Phi^{i} d x^{j}-\Phi^{i} d x^{i}}
$$

where the two indices $i$ and $j$ with $i \neq j$, are held fixed, and $l \neq i, j$ is allowed to vary over the remaining integers 1 to $n$.

If $n=2$ or if $n \geqq 3$, that is, if $n \geqq 2$, then under $T$, we must have the 
identity

$$
\begin{gathered}
\frac{3\left(\Phi^{i} \bar{\Phi}^{j}-\bar{\Phi}^{i} \Phi^{j}\right)}{\left(\bar{\Phi}^{i} d x^{j}-\bar{\Phi}^{j} d x^{i}\right)\left(\Phi^{i} d x^{j}-\Phi^{i} d x^{i}\right)} \\
=\left(\frac{2 k}{1+k}\right)\left[\frac{\bar{g}_{\alpha \beta} \bar{\Phi}^{\alpha} d x^{\beta}}{\left(\bar{\Phi}^{i} d x^{i}-\bar{\Phi}^{i} d x^{i}\right)\left(\bar{g}_{\alpha \beta} d x^{\alpha} d x^{\beta}\right)}-\frac{g_{\alpha \beta} \Phi^{\beta} d x^{\alpha}}{\left(\Phi^{i} d x^{j}-\Phi^{j} d x^{i}\left(g_{\alpha \beta} d x^{\alpha} d x^{\beta}\right)\right.}\right],
\end{gathered}
$$

and also the identity

$$
\begin{aligned}
& \frac{\frac{d \bar{\Phi}^{i}}{d x^{j}}-\frac{d \bar{\Phi}^{j}}{d x^{j}} \frac{d x^{i}}{d x^{j}}}{\bar{\Phi}^{i}-\bar{\Phi}^{i} \frac{d x^{i}}{d x^{j}}}+2 \bar{\Gamma}_{\alpha \beta}^{j} \frac{d x^{\alpha}}{d x^{j}} \frac{d x^{\beta}}{d x^{j}}+\frac{\bar{\Phi}^{j}}{\bar{\Phi}^{i}-\bar{\Phi}^{j} \frac{d x^{i}}{d x^{j}}}\left[\bar{\Delta}^{i}-\frac{d^{2} x^{i}}{d\left(x^{i}\right)^{2}}\right] \\
& =\frac{\frac{d \Phi^{i}}{d x^{j}}-\frac{d \Phi^{j}}{d x^{j}} \frac{d x^{i}}{d x^{j}}}{\Phi^{i}-\Phi^{j} \frac{d x^{i}}{d x^{j}}}+2 \Gamma_{\alpha \beta}^{j} \frac{d x^{\alpha}}{d x^{j}} \frac{d x^{\beta}}{d x^{j}}+\frac{\Phi^{j}}{\Phi^{i}-\Phi^{j} \frac{d x^{i}}{d x^{j}}}\left[\Delta^{i}-\frac{d^{2} x^{i}}{d\left(x^{j}\right)^{2}}\right],
\end{aligned}
$$

where the two indices $i$ and $j$ with $i \neq j$, are fixed.

Therefore the required point correspondeuce $T$ is a projective transformation $T$ between the two Riemannian spaces $V_{n}$ and $\bar{V}_{n}$, such that it obeys the identities (23.1), (23.2), and (23.3).

When $n=2$, the two distinct indices $i$ and $j$ may be taken as $i=1$, $j=2$. If

$$
U=g_{11} d x^{1}+g_{12} d x^{2}, \quad V=g_{12} d x^{1}+g_{22} d x^{2}
$$

$$
\bar{U}=\bar{g}_{11} d x^{2}+\bar{g}_{12} d x^{2}, \quad \bar{V}=\bar{g}_{12} d x^{1}+\bar{g}_{22} d x^{2}
$$

the identity (23.2) can be written in the form

$$
\begin{aligned}
& \left(\frac{3+k}{1+k}\right)\left(\Phi^{1} \bar{\Phi}^{2}-\bar{\Phi}^{1} \Phi^{2}\right)\left(U d x^{1}+V d x^{2}\right)\left(\bar{U} d x^{1}+\bar{V} d x^{2}\right) \\
= & \left(\frac{2 k}{1+k}\right)\left(\Phi^{1} d x^{2}-\Phi^{2} d x^{1}\right)\left(\bar{\Phi}^{1} d x^{2}-\bar{\Phi}^{2} d x^{1}\right)(\bar{U} V-U \bar{V}) .
\end{aligned}
$$

Since $k \neq-1,0$, it is deduced that this identity is ralid if and only if either $k \neq-3, \Phi^{1} \bar{\Phi}^{2}-\bar{\Phi}^{1} \Phi^{2}=0, \bar{U} V-U \bar{V}=0$, or $k=-3, \bar{U} V-U \bar{V}=\overline{0}$. 
For either case, this states that $T$ is a conformal map when $n=2$. Consequently $T$ is a homothetic transformation in the case of $n=2$ dimensions.

The following proposition is an extension of the above result to $n$ dimensions.

THeOREM 23.1. - A point correspondence $T$ is a homothetic transformation $T$ between two Riemannian spaces $\bar{\nabla}_{n}$ and $\bar{V}_{n}$, if and only if it converts every complete physical system $S_{k}$ with $k \neq-1$, 0 , of $\infty^{2 n-1}$ trajectories $C$ in $\nabla_{n}$, into a complete physical system $\overline{S_{k}}$ with the same $k \neq-1,0$, of $\infty^{2 n-1}$ trajec. tories $\bar{C}$ in $\bar{V}_{n}$.

When $k$ is infinite, this includes a corresponding result concerning com. plete velocity systems $S_{\infty}$ of $\infty^{2 n-1}$ velocity curves $C$.

The above proposition has been proved when $n=2$. It only remains to prove it for the case when $n \geqq 3$.

From the identities (23.1), there exists a scalar point function $\rho=\rho(x) \neq 0$, which is never zero and at least of class two in an $n$ dimensional region of points $x$, such that

$$
\vec{\Phi}^{i}=\rho \Phi^{i},
$$

for $i=1,2, \ldots, n$.

Substituting (23.6) into the identity (23.2), it becomes the identity

$$
\frac{\left.\overline{(g}_{\alpha \beta} \Phi^{\alpha} d x^{\beta}\right)}{\left(\bar{g}_{\alpha \beta} d x^{\alpha} d x^{\beta}\right)}=\frac{\left(g_{\alpha \beta} \Phi^{\alpha} d x^{\beta}\right)}{\left(g_{\alpha \beta} d x^{\alpha} d x^{\beta}\right)},
$$

since $k \neq-1,0$. Of course, $k$ may be infinite.

Equating the corresponding coefficients of $\Phi^{*}$ in the above identity, it is found that

$$
\frac{\bar{g}_{i j} d x^{j}}{\left(\bar{g}_{\alpha \beta} d x^{\alpha} d x^{\beta}\right)}=\frac{g^{i j} d x^{j}}{\left(g_{\alpha \beta} d x^{\alpha} d x^{\beta}\right)}
$$

for $i=1,2, \ldots, n$.

Upon equating corresponding coefficients of the $d x^{i}$ in the identities (23.8), it is found that there exists a positive scalar point function $\mu=\mu(x)>0$, of at least class three in the $n$ dimensional region of points $x$, snch that

$$
d \bar{s}=\mu d s, \text { or } \bar{g}_{i j}=\mu^{2} g_{i j} .
$$

Therefore, $T$ is necessarily a conformal map.

Since $T$ is both a projective correspondence and a conformal correspondence, it follows that $T$ is a homothetic transformation $T$ between the two Riemannian spaces $V_{n}$ and $\bar{V}_{n}$. This means that $\mu>0$, is a positive constant. 
Substituting (23.6) and (23.9) where $\mu>0$, is a positive constant, into the identity (23 3), it is found that $\rho \neq 0$, is a non-zero constant.

It follows that under these hypotheses, the identities (23.1), (23.2), and (23.3), are satisfied.

Consequently, the proof of Theorem 23.1, is complete.

\section{REFERENCES}

[1] Kasner and DE Crcco, Geometrical properties of physical curves in space of $n$ dimensions, Revists de Matematica y Fisica Teorica de la Universidad Nacional del Tucuman, (Argentina), 8, 127.137, 1951.

[2] These were studied originally by KASNER in the Euclidean plane. See his Princeton Colloquium Lectures, pages 8 and 9.

[3] Eisenhart, Riemannian geometry, Princeton University Press 1949.

[4] WhIтTAKER, A treatise on the analytical dynamics of particles and rigid bodies, Fourth edition, Dover Publications, 1944.

[5] De Cioco, Conservative physical families of curves on a surface, Revists de « Matematica $y$ Fisica Teorica de la Universidad Nacional del Tucuman », (Argentina), 9, 23.36, 1952.

[6] KASNER, Differential equations of the type; $y^{\prime \prime \prime}=G y^{\prime \prime}+H y^{\prime 2}$, Proceedings of the National Academy of Sciences, 28, 333-338, 1942.

[7] Kasner and Mittleman, A general theorem of the initial curvature of dynamical trajectories, Proceedings of the National Academy of Sciences, 28, 48-52, 1942.

[8] KASNER and De CICCo, A generalized theory of dynamical trajectories, Transactions of the "American Mathematical Society ", 54, 23-38, 1943.

[9] KASNer, Dynamical trajectories and curvature trajectories, Bulletin of the American Mathematical Society ", 40, 449.455, 1934.

[10] For an extension of this theorem of KASNER to space, see De Cicco, Dynamical and curvature trajectories in space, Transactions of the American Society, 57, 270-286, 1945.

[11] KASNer and DE Crcco, The geometry of velocity systems, Bulletin of the American Mathematical Society, 49, 236-245», 1943.

[12] KASNer, Natural families of trajectories: Conservative fields of force, Transactions of the "American Mathematical Society , 10, 201-219, 1909. Also see KASNer, The thenrem of Thompson and Tait, and naturat families of trajectories, Transactions of the *American Mathematical Society ", 11, 121-140, 1910.

[13] KASNER and DE CICCO, Physical families in conservative fields of force, Proceedings of the "National Academy of Sciences, 35, 419-422, 1949.

[14] Kasner, The infinitesimal contact transformations of mechanics, "Bulletin of the American Mathematical Society, 16, 408-412, 1910.

[15] EisenhaRT, Continuous groups of transformations, "Princeion University Press, 1933..

[16] DE Cicco, New proofs of the theorems of KASNER concerning the infinitesimal contact transformations of mechanics, «Journal of Mathematics and Physics, 26, 104-109», 1947.

[17] Ersenhart, Non.Riemannian geometry, "American Mathematical Society" Colloquium Publications, 1927.

[18] KaSNer and DE Croco, Generaliaation of Appell's transformation, «Journal of Mathematies and Physies», 27, 262-269, 1949.

[19] - -, Transformation theory of physical curves, Proceedings of the "National Aca. demy of Sciences, 33, 338.342, 1947. 